\title{
ANALYTIC SOLUTIONS OF CONVOLUTION EQUATIONS ON CONVEX SETS WITH AN OBSTACLE IN THE BOUNDARY
}

\author{
S. N. MELIKHOV and SIEGFRIED MOMM
}

\section{Introduction}

Let $Q \subset \mathrm{C}^{N}$ be convex and not pluripolar. More than 30 years ago, Martineau [14] investigated the spaces $A(Q)$ of analytic functions on $Q$, in the case that $Q$ admits a countable fundamental system of compact subsets. These spaces can also be described in the following way: $Q$ is locally closed, i.e. $Q$ is the union of its relative interior $\operatorname{int}_{r} Q$ and an open portion $\omega$ of its relative boundary $\partial_{r} Q$, and $A(Q)$ consists of all functions analytic on $\operatorname{int}_{r} Q$ having an analytic extension to some neighborhood of $\omega$. In this sense, $\partial_{r} Q \backslash \omega$ can be considered as an obstacle for the analytic continuation of $f$.

This setup covers in particular convex domains $Q$ in $\mathrm{C}^{N}$, nonpluripolar convex compact sets $Q$ in $\mathrm{C}^{N}$, and convex domains $Q$ in $\mathrm{R}^{N}$.

If $P=\sum_{\alpha \in \mathrm{N}_{0}^{N}} a_{\alpha} z^{\alpha}$ is a nonzero entire function on $\mathrm{C}^{N}$ of at most order one and zero type, in the more general setting of convolution equations, we consider the partial differential equation

$$
P(D) f=\sum_{\alpha \in \mathrm{N}_{0}^{N}} a_{\alpha} f^{(\alpha)}=g, \quad g, f \in A(Q) .
$$

By the assumption on $P$, we know that $P(D) f \in A(Q)$ for each $f \in A(Q)$. If $Q$ is open in $\mathrm{C}^{N}$, by an old result of Martineau [15], which for $N=1$ has been obtained independently also by Korobeĭnik [4], the differential operator $P(D): A(Q) \rightarrow A(Q)$ is surjective for every $P$. If $Q$ is open in $\mathrm{R}^{N}$, Hörmander [2] characterized the surjective operators $P(D): A(Q) \rightarrow A(Q)$ of finite order. For general $Q$, only in the case $N=1$, it is well understood, under which additional condition on the pair $(P, Q)$, the differential operator $P(D)$ : $A(Q) \rightarrow A(Q)$ is surjective (Napalkov and Rudakov [30], Korobeĭnik [5], Maltsev [13]).

As a consequence of these results, for $N=1$ the operator $P(D)$ : 
$A(Q) \rightarrow A(Q)$ is surjective for all $P$ if and only if $Q \subset \mathrm{C}$ has a fundamental system of convex neighborhoods. A geometric characterization of this property is that $Q$ is strictly convex at $\partial_{r} \omega$, i.e. the intersection of $Q$ and each supporting line (= hyperplane in general) to $Q$ is compact. Of course the strict convexity of $Q$ at $\partial_{r} \omega$ is also for $N \geq 1$ sufficient for $P(D): A(Q) \rightarrow A(Q)$ being surjective for all $P$. It is very likely that this property is necessary also for $N \geq 1$ (see Prop. 1.16).

In the present paper, for surjective operators $P(D): A(Q) \rightarrow A(Q)$, we investigate whether there are more explicit solutions $f=R(g) \in A(Q)$ of the equation $P(D) f=g \in A(Q)$, given by a "formula" $R$. To be precise, we look for a continuous linear mapping $R: A(Q) \rightarrow A(Q)$ with $P(D) \circ R=\operatorname{id}_{A(Q)}$. This question makes sense if there is a unique natural topology on the vector space $A(Q)$. Martineau [14] gave sufficient conditions for the uniqueness of this topology, which apply to all examples which have been mentioned up to now and to those $Q$ which are strictly convex at $\partial_{r} \omega$. In view of very recent results of Vogt [35] and Wengenroth [36], [37], the uniqueness of the topology of $A(Q)$ happens to be fulfilled precisely for those sets $Q$ for which a certain machinery can be applied to characterize the surjective operators $P(D): A(Q) \rightarrow A(Q)$.

Our results on the existence of continuous linear right inverses will be given in terms of certain functions $C_{H}^{\infty}, C_{H}^{0}$ defined on the unit sphere $S$ of $\mathrm{C}^{N}$ and which are known to describe the boundary behavior of the pluricomplex Green functions of the interior of $Q$ and of the complement of the closure of $Q$, respectively (see [25] and [27]). To give their definition we assume that $Q$ is bounded, has interior points and that 0 is one of them. $H: \mathrm{C}^{N} \rightarrow[0, \infty[$ denotes the supporting function of the closure of $Q$, i.e. the positively homogeneous function $H(z):=\sup _{w \in Q} \operatorname{Re}\langle w, z\rangle(\langle\cdot, \cdot\rangle$ being the scalar product in $\left.\mathrm{C}^{N}\right)$. By $v_{H}^{\infty}$ and $v_{H}^{0}$, we denote the largest plurisubharmonic functions which are at the same time bounded by $H(z)$ for all $z \in \mathrm{C}^{N}$ and by $\log |z|$ as $|z| \rightarrow \infty$ or as $|z| \rightarrow 0$, respectively. There are unique functions $\left.\left.C_{H}^{\infty}: S \rightarrow\right] 0, \infty\right]$ and $C_{H}^{0}: S \rightarrow[0, \infty[$ defined by

$$
\left\{z \in \mathrm{C}^{N} \mid v_{H}^{\infty}(z)=H(z)\right\}=\left\{\lambda a \mid a \in S, 0 \leq \lambda \leq 1 / C_{H}^{\infty}(a)\right\}
$$

and

$$
\left\{z \in \mathrm{C}^{N} \mid v_{H}^{0}(z)=H(z)\right\}=\left\{\lambda a \mid a \in S, 1 / C_{H}^{0}(a) \leq \lambda<\infty\right\} .
$$

By $S_{\omega}$, we denote the set of all directions $a \in S$ such that the supporting hyperplane $\operatorname{Re}\langle z, a\rangle=H(a)$ intersects $Q$ (in some point of $\omega$ ). Strict convexity of $Q$ at $\partial_{r} \omega$ implies that $S_{\omega}$ is open in $S$.

THEOREM I. If $Q \subset \mathrm{C}^{N}$ is strictly convex at $\partial_{r} \omega$, the following are equivalent: 
(i) Each nonzero $P(D): A(Q) \rightarrow A(Q)$ admits a continuous linear right inverse $R$.

(ii) After a unitary transformation of $\mathrm{C}^{N}, Q$ equals the Cartesian product $Q^{\prime} \times \mathrm{C}^{N^{\prime \prime}}$, where $Q^{\prime} \subset \mathrm{C}^{N^{\prime}}$ is bounded, with nonempty interior, such that $P^{\prime}(D): A\left(Q^{\prime}\right) \rightarrow A\left(Q^{\prime}\right)$ admits a continuous linear right inverse for each nonzero $P^{\prime}$ of $N^{\prime}$ variables, and such that either $Q=Q^{\prime}$ or $Q$ is open.

For this reason it suffices to consider bounded $Q$ with nonempty interior:

TheOREM II. Let $Q \subset \mathrm{C}^{N}$ be strictly convex at $\partial_{r} \omega$, bounded, and with 0 in its nonempty interior. Then the following are equivalent:

(i) Each nonzero $P(D): A(Q) \rightarrow A(Q)$ admits a continuous linear right inverse $R$.

(ii) $C_{H}^{\infty}$ is bounded on some neighborhood of $S \backslash S_{\omega}$, and $1 / C_{H}^{0}$ is bounded on each compact subset of $S_{\omega}$.

These equivalent conditions are fulfilled if $\partial Q$ is of Hölder class $C^{1, \lambda}$ for some $\lambda>0$. They are not fulfilled for polyhedra $Q$ ([28]).

Furthermore, in the case $N=1$ of one complex variable, we characterize whether a single operator $P(D): A(Q) \rightarrow A(Q)$ admits a continuous linear right inverse.

Our results on the existence of right inverses extend results of Schwerdtfeger [33], Taylor [34], Meise and Taylor [16] for $Q=\mathrm{C}^{N}$, of [20], [23], [22], [17], Korobeunik and Melikhov [8] for open or compact convex sets in $C^{N}$, and of Langenbruch [12] and Korobeunik [6], [7] for intervals of $\mathrm{R}$ and for polygons in $\mathrm{C}$, respectively. Since the locally convex spaces $A(Q)$ have a rather complicated structure - in general as in the cited results on intervals and polygons, they are neither Fréchet spaces nor strong duals of Fréchet spaces the procedure of proof of most of the former work does not apply. For the results on intervals and polygons, ad hoc arguments have been used which are limited to the special situation (in fact, part of the proof is a reduction to corresponding problems for inscribed or circumscribed open or closed polyhedra or discs). Our approach consists in extending the technique used in [22] for open $Q$ and in [17] for compact $Q$, which (for the constructive direction of the results) applies a continuous linear right inverse of the $\bar{\partial}$-operator acting on certain auxiliary spaces.

Our paper is organized as follows. In the first chapter we introduce the spaces and operators of our interest, and we prove the announced result on the surjectivity. In the second chapter we obtain an abstract criterion for the existence of solution operators in terms of the existence of certain plurisubharmonic functions. Chapter 3 is devoted to the evaluation of the abstract condition which proves in particular the Theorems I and II. In chapter 
4 we improve the results of chapters 2 and 3 in the special case of one complex variable.

ACKNOWLEDGEMENT. The first named author thanks for the support by the Deutscher Akademischer Austauschdienst and by the Russian Foundation of Fundamental Research. The second author thanks the Erwin Schrödinger Institut for its hospitality during his stay in Vienna in February 1995.

\section{Preliminaries}

1.1. Notations. If $B \subset \mathrm{C}^{N}$, by $\operatorname{cv}(B), \mathrm{cl} B$, and $\operatorname{int} B$ we will denote the convex hull, the closure, and the interior of $B$, respectively. By int ${ }_{r} B, \partial_{r} B$ we sometimes denote the relative interior and the relative boundary of $B$ with respect to a certain larger set (which is determined by the context). For notions from convex analysis, we refer to Schneider [32].

1.2. Lemma. A convex set $Q \subset \mathrm{C}^{N}$ admits a countable fundamental system of compact sets if and only if $Q$ is the union of the relative interior $\operatorname{int}_{r} Q$ of $Q$ and an open portion $\omega$ of the relative boundary $\partial_{r} Q$ of $Q$. In this case, if $\left(K_{n}\right)_{n \in \mathrm{N}}$ and $\left(\omega_{n}\right)_{n \in \mathrm{N}}$ are fundamental systems of compact sets for $\mathrm{int}_{r} Q$ and of $\omega$, respectively, the convex hulls $Q_{n}:=\operatorname{cv}\left(K_{n} \cup \omega_{n}\right), n \in \mathrm{N}$, define a fundamental system of compact subsets of $Q$.

Proof. If $\left(Q_{n}\right)_{n \in \mathrm{N}}$ is a fundamental system of compact convex sets, then $Q=\bigcup_{n \in \mathrm{N}} Q_{n}=\operatorname{int}_{r} Q \cup \omega$ with $\omega:=Q \backslash \operatorname{int}_{r} Q \subset \partial_{r} Q$. Assume that $\omega$ is not relatively open in $\partial_{r} Q$. Then there are $w_{0} \in \omega$ and a sequence $\left(z_{n}\right)_{n \in \mathbb{N}}$ in $\partial_{r} Q \backslash \omega$ converging to $w_{0}$. For $n \in \mathrm{N}$, therefore $z_{n} \notin Q$ and thus $z_{n} \notin Q_{n}$. Since $Q_{n}$ is compact, we can choose $w_{n} \in Q$ with $\left|z_{n}-w_{n}\right|<\min \left\{\operatorname{dist}\left(z_{n}, Q_{n}\right) /\right.$ $2,1 / n\}$. By this choice, $A:=\left\{w_{j} \mid j=0,1, \ldots\right\}$ is a compact subset of $Q$ which is not contained in any $Q_{n}, n \in \mathrm{N}$. This is a contradiction.

Vice versa, $Q_{n}=\operatorname{cv}\left(K_{n} \cup \omega_{n}\right),\left(Q_{n}\right)_{n \in \mathrm{N}}$, is a fundamental system of compact sets: Let $K$ be a compact subset of $Q$. Then $\kappa:=K \cap \partial_{r} Q$ is a compact subset of $\omega$ and thus contained in some $\omega_{n}$. Fix a point $w_{0} \in K_{1}$. If $p>n$ is chosen such that $\omega_{n} \subset \operatorname{int}_{r} \omega_{p}$, the set $U:=\operatorname{cv}\left(\left\{w_{0}\right\} \cup \omega_{p}\right)$ is a neighborhood of $\kappa$ in $Q$. Hence $K^{\prime}:=K \backslash U$ is relatively compact in $\operatorname{int}_{r} Q$ and is thus contained in some $K_{m}, m \geq p$. This gives

$$
K \subset K_{m} \cup U \subset \operatorname{cv}\left(K_{m} \cup \omega_{m}\right) .
$$

Remark. Of course, also $\tilde{Q}_{n}:=\left\{z \in \operatorname{cl} Q \mid \operatorname{dist}\left(z, \partial_{r} Q \backslash \omega\right) \geq 1 / n\right.$ and $\left.|z| \leq n\right\}$, $n \in \mathrm{N}$, is a fundamental system of compact subsets of $Q$.

1.3. Definition and Remark. A convex set $Q \subset \mathrm{C}^{N}$ admitting a countable fundamental system $\left(Q_{n}\right)_{n \in \mathrm{N}}$ of compact subsets of $Q$ is called locally closed (since - by Lemma 1.2 - it is locally closed in the affine hull of $Q$ ). We will 
write $\omega:=Q \cap \partial_{r} Q$, where $\partial_{r} Q$ denotes the relative boundary of $Q$ in its affine hull. We may of course assume that the sets $Q_{n}$ are convex and that $Q_{n} \subset Q_{n+1}$.

$Q \subset \mathrm{C}^{N}$ will be called (C-) strictly convex at $\partial_{r} \omega$ if the intersection of $Q$ with each supporting (complex) hyperplane to $\mathrm{cl} Q \subset \mathrm{C}^{N}$ is compact.

If int $Q=\emptyset$, by our definition, $Q \subset \mathrm{C}^{N}$ is strictly convex at $\partial_{r} \omega$ if and only if $Q$ is compact. If int $Q \neq \emptyset, Q$ is (C-) strictly convex at $\partial_{r} \omega$ if and only if each line segment (of which the $\mathrm{C}$-linear affine hull belongs to some supporting hyperplane to $\operatorname{cl} Q$ ) of $\omega=Q \cap \partial Q$ is relatively compact in $\omega$.

1.4. Convention. In the sequel, $Q$ will be a nonpluripolar, locally closed, convex subset of $\mathrm{C}^{N}$ with fundamental system of compact convex sets $Q_{n} \subset Q_{n+1}, n \in \mathrm{N}$. According to Lemma 1.2 we write $Q=\operatorname{int}_{r} Q \cup \omega$. $\left(\omega_{n}\right)_{n \in \mathrm{N}}$ will denote some fundamental system of compact subsets of $\omega=Q \cap \partial_{r} Q$.

$K \subset \mathrm{C}^{N}$ will always denote a compact convex set. We assume that also $Q+K$ is locally closed and that $\left(Q_{n}+K\right)_{n \in \mathrm{N}}$ is a fundamental system of compact subsets of $Q+K$.

1.5. ReMARK. (a) If $N=1$ or if $Q$ is strictly convex at $\partial_{r} \omega$, then for each compact convex set $K \subset \mathrm{C}^{N}$, also $Q+K$ is locally closed, and $Q_{n}+K$ is a fundamental system of compact subsets.

Proof. Assume that $Q_{n}+K, n \in \mathrm{N}$, is not fundamental. Then there are sequences $q_{n} \in Q, p_{n} \in K$ converging to $q \in \bar{Q}$ and $p \in K$ respectively, such that $z_{n}:=q_{n}+p_{n}$ converge to $z \in Q+K$, but $\left\{z_{n} \mid n \in \mathrm{N}\right\} \cup\{z\}$ is not contained in some $Q_{n}+K$. If $z=q_{0}+p_{0}$ with $q_{0} \in Q$ and $p_{0} \in K$ it follows that $q \notin Q,\left[q, q_{0}\right] \subset \partial_{r} Q$, and $\left[p_{0}, p\right] \subset \partial K$. This is a contradiction if $Q$ is strictly convex at $\partial_{r} \omega$. If $N=1$, we obtain that the relative boundary of $Q+K$ near $z=q_{0}+p_{0}$ is an interval belonging to $Q+K$ which again implies a contradiction to our assumption.

(b) In general $Q+K$ is not locally closed again: Let $Q \subset \mathrm{R}^{3}$ be the convex hull of the unit disc $D$ in $R^{2} \times\{0\}$ and the points $(0, \pm 1,1)$ minus the "bottom" $D$. Then $Q$ is locally closed, but $Q+K$ is not locally closed for $K:=\{(0,0)\} \times[-1,0]$ (consider the point $(0,1,0)$ for example).

1.6. Notations. By $U$, we denote the open unit ball of $\mathrm{C}^{N}$. For each convex set $D \subset \mathrm{C}^{N}$ let $H_{D}: \mathrm{C}^{N} \rightarrow \mathrm{R} \cup\{\infty\}$ denote the support function of $D$

$$
H_{D}(z):=\sup _{w \in D} \operatorname{Re}\langle z, w\rangle, \quad z \in \mathrm{C}^{N} .
$$

Here $\langle z, w\rangle:=\sum_{j=1}^{N} z_{j} \bar{w}_{j}$. We put $H_{n}:=H_{Q_{n}}, n \in \mathrm{N}$. The support function of $Q$ and $K$ will be denoted by $H$ and $L$ respectively. If $u$ is a function on a subset of $\mathrm{C}^{N}$, we shall write $\bar{u}$ for the function $\bar{u}(z):=u(\bar{z})$. 
1.7. Definition. For all $n, m \in \mathrm{N}$ let $A_{n, m}$ denote the Banach space of all entire functions $f$ on $\mathrm{C}^{N}$ satisfying

$$
\|f\|_{n, m}:=\sup _{z \in \mathrm{C}^{N}}|f(z)| \exp \left(-H_{n}(\bar{z})-|z| / m\right)<\infty .
$$

We consider the Fréchet spaces $A_{\bar{H}_{n}}^{0}:=\operatorname{proj}_{m \rightarrow \infty} A_{n, m}, n \in \mathrm{N}$, and the (LF)space

$$
A_{\bar{H}}:=\operatorname{ind}_{n \rightarrow \infty} A_{\bar{H}_{n}}^{0} .
$$

1.8. Definition. For all $n, m \in \mathrm{N}$ let $E_{n, m}:=A^{\infty}\left(Q_{n}+\frac{1}{m} U\right)$ denote the Banach space of all bounded holomorphic functions on the open set $Q_{n}+\frac{1}{m} U$, equipped with the norm

$$
|f|_{n, m}:=\sup _{z \in Q_{n}+\frac{1}{m} U}|f(z)| .
$$

We consider the spaces $A\left(Q_{n}\right)=\cup_{m \in \mathrm{N}} E_{n, m}$ of all functions holomorphic in some neighborhood of $Q_{n}, n \in \mathrm{N}$, and endow them with their natural inductive limit topology. If $A(Q)$ is the vector space of all functions which are holomorphic on some neighborhood of $Q$, we have $A(Q)=\bigcap_{n \in \mathrm{N}} A\left(Q_{n}\right)$, and we endow this vector space with the topology of

$$
A(Q):=\operatorname{proj}_{n \rightarrow \infty} A\left(Q_{n}\right)=\operatorname{proj}_{n \rightarrow \infty} \operatorname{ind}_{m \rightarrow \infty} A^{\infty}\left(Q_{n}+\frac{1}{m} U\right) .
$$

This topology does not depend on the choice of the fundamental system of compact sets.

1.9. REMARK. (a) There is another reasonable choice for a topology on $A(Q)$, namely the topology of the inductive limit $\operatorname{ind}_{\Omega} A(\Omega)$, where $\Omega$ runs over all open neighborhoods of $Q$, and where $A(\Omega)$ denotes the Frechet space of all analytic functions on $\Omega$. However, in order that the question posed in 1.11 makes sense, we are mainly interested in those sets $Q$ for which these two reasonable topologies coincide, i.e. for which there is one natural topology on $A(Q)$. This is the case if and only if the locally convex space $A(Q)$ defined in Definition 1.8 is ultrabornological (here we note that - because of the completeness of $A(Q)$ - this space is ultrabornological if and only if it is bornological).

(b) By Martineau [14], Thm. 1.2, $A(Q)$ is ultrabornological for example in the following three cases: If $N=1$, if $Q \subset \mathrm{R}^{N}$, or if $Q \subset \mathrm{C}^{N}$ is C-strictly convex at $\partial_{r} \omega$ with nonempty interior. In particular, $A(Q)$ is ultrabornological if $Q$ is strictly convex at $\partial_{r} \omega$.

Proof of (a): The inductive limit topology is of course bornological. Vice 
versa, à priori the inductive limit topology is finer than the topology of $A(Q)$.

The straight forward proof of " $A(Q) \supset \cap_{n \in \mathrm{N}} A\left(Q_{n}\right)$ " (which has been omitted in 1.8) shows that each bounded subset of $A(Q)$ is bounded in the inductive limit (see Martineau [14], Prop. 1.2). (If $B$ is bounded in $A(Q)$, it is bounded in each step $A\left(Q_{n}\right)$ of the projective limit. Since $A\left(Q_{n}\right)$ is a (DFS)space, $B$ is bounded in some step $E_{n, m}$ of the inductive limit $A\left(Q_{n}\right)$. Then the proof of " $A(Q) \supset \cap_{n \in \mathrm{N}} A\left(Q_{n}\right)$ " of 1.8 shows that $B$ is bounded on each compact subset of some $\Omega$, i.e. $B$ is bounded in some $A(\Omega)$.) Since $A(Q)$ is now assumed to be bornological, the identity map from $A(Q)$ into the inductive limit is continuous. This follows also from Martineau [14], Prop. 1.7.

1.10. Lemma. For all $n, m \in \mathrm{N}$ let $|\cdot|_{n, m}^{*}$ denote the norm of the dual Banach space $E_{n, m}^{\prime}$. Then the $(L F)$-spaces $A(Q)^{\prime}:=\operatorname{ind}_{n \rightarrow \infty} A\left(Q_{n}\right)^{\prime}$ and $A_{\bar{H}}$ are isomorphic by the Laplace transformation

$$
\mathscr{F}: A(Q)^{\prime} \rightarrow A_{\bar{H}}, \quad \widetilde{F}(\mu)(z):=\hat{\mu}(z):=\mu(\exp (\cdot z)), \quad z \in \mathrm{C}^{N},
$$

$(w \cdot z:=\langle w, \bar{z}\rangle)$. Moreover for all $n, m \in \mathrm{N}$ there is $C>0$ with:

(i) $\|\mathscr{F}(\mu)\|_{n, m} \leq|\mu|_{n, m}^{*}$ for all $\mu \in A\left(Q_{n}\right)^{\prime}$;

(ii) $\left|\mathscr{F}^{-1}(f)\right|_{n, m}^{*} \leq C\|f\|_{n, m+1}$ for all $f \in A_{H_{n}}^{0}$.

Proof. Since the linear span of the exponentials $w \mapsto e^{w \cdot z}, z \in \mathrm{C}^{N}$, is dense in $A\left(Q_{n}\right), A(Q)$ is dense in each step $A\left(Q_{n}\right), n \in \mathrm{N}$. Hence $A(Q)^{\prime}=\operatorname{ind}_{n \rightarrow \infty} A\left(Q_{n}\right)^{\prime}$ holds algebraically, and the assertion follows from the well known corresponding one for the spaces $A\left(Q_{n}\right)^{\prime}, n \in \mathrm{N}$ (see Hörmander [1], Thm. 4.5.3, and see also [17], 1.4).

Remark. It follows from Schaefer [31], Ch. IV, 4.4, that the (LF)-space topology of $A(Q)^{\prime}$ in fact equals the strong topology. However, we will not apply this fact.

1.11. Definition. For $\mu \in A(K)^{\prime}$ we define the convolution operator $T_{\mu}: A(Q+K) \rightarrow A(Q)$ by

$$
T_{\mu}(f)(z):=\mu(f(z+\cdot)), \quad z \in Q .
$$

$T_{\mu}$ is a continuous linear map, since $T_{\mu}: A\left(Q_{n}+K\right) \rightarrow A\left(Q_{n}\right)$ is continuous for each $n$ (see [17]). If $K=\{0\}$ we write $P(z):=\hat{\mu}(z)=\sum_{\alpha \in \mathrm{N}_{0}^{N}} a_{\alpha} z^{\alpha}$ and get $P(D) f:=\sum_{\alpha \in \mathrm{N}_{0}^{N}} a_{\alpha} f^{(\alpha)}=T_{\mu}(f)$.

In the present article we look whether for a given surjective operators $T_{\mu}$ there is a continuous linear right inverse $R$ for $T_{\mu}$, i.e. a continuous linear mapping $R: A(Q) \rightarrow A(Q+K)$ with $T_{\mu} \circ R=\operatorname{id}_{A(Q)}$. By Lemma 1.10, the dual map $T_{\mu}^{\prime}: A(Q)^{\prime} \rightarrow A(Q+K)^{\prime}$ can be identified with the multiplication operator $M_{\hat{\mu}}: A_{\bar{H}} \rightarrow A_{\bar{H}+\bar{L}}, f \mapsto \hat{\mu} \cdot f$. 
1.12. Lemma. The following assertions are equivalent for a surjective convolution operator $A:=T_{\mu}: A(Q+K) \rightarrow A(Q)$ :

(i) $A$ has a continuous linear right inverse

(ii) $A^{\prime}$ has a continuous linear left inverse

(iii) $\operatorname{im} A^{\prime}$ is closed and the quotient map $q: A(Q+K)^{\prime} \rightarrow A(Q+K)^{\prime} / \operatorname{im} A^{\prime}$ has a continuous linear right inverse.

Proof. (i) $\Rightarrow$ (ii) The dual map of the continuous linear right inverse of $A$ is a continuous linear left inverse for $A^{\prime}$.

(ii) $\Rightarrow$ (i): We recall that $A(Q)^{\prime}:=\operatorname{ind}_{n} A\left(Q_{n}\right)^{\prime}$ and $A(Q+K)^{\prime}:=$ $\operatorname{ind}_{n} A\left(Q_{n}+K\right)^{\prime}$ (see Lemma 1.10). If $P: A(Q+K)^{\prime} \rightarrow A(Q)^{\prime}$ is a continuous linear left inverse for $A^{\prime}$, by Grothendieck's factorization theorem, for each $n$ there is $n^{\prime}$ such that the induced maps $P_{n}: A\left(Q_{n}+K\right)^{\prime} \rightarrow A\left(Q_{n^{\prime}}\right)^{\prime}$ are well defined and continuous. Since the (DFS)-spaces $A\left(Q_{n^{\prime}}\right)$ and $A\left(Q_{n}+K\right)$ are reflexive, the dual maps $P_{n}^{\prime}: A\left(Q_{n^{\prime}}\right) \rightarrow A\left(Q_{n}+K\right)$ induce a continuous linear map $R: A(Q) \rightarrow A(Q+K)$ and $(A \circ R)^{\prime}=P \circ A^{\prime}=\mathrm{id}$. Thus $R=P^{\prime}$ is a continuous right inverse for $A$.

(ii) $\Leftrightarrow$ (iii): If $P: A(Q+K)^{\prime} \rightarrow A(Q)^{\prime}$ is a continuous left inverse for $A^{\prime}$, i.e. $P \circ A^{\prime}=\operatorname{id}_{A(Q)^{\prime}}$, then $Q:=A^{\prime} \circ P$ is a continuous projection onto $\operatorname{im} A^{\prime}$. Hence $\operatorname{im} A^{\prime}$ is closed and $R: A(Q+K)^{\prime} / \operatorname{im} A^{\prime} \rightarrow A(Q+K)^{\prime}$ defined by $R(q(\nu)):=\nu-Q(\nu)$ is a continuous linear right inverse for $q$.

Vice versa, if $R$ is a continuous linear right inverse for $q$, then $I-R \circ q$ is a continuous projection onto $\operatorname{ker}(R \circ q)=\operatorname{im} A^{\prime}$. Thus $P:=A^{\prime-1} \circ(I-R \circ q)$ is a linear left inverse for $A^{\prime}$. It is continuous by the closed graph theorem for (LF)-spaces (see Langenbruch [12] for this reasoning).

1.13. Definition. Let $\mu \in A(K)^{\prime}$. If $A \subset S:=\left\{z \in \mathrm{C}^{N}|| z \mid=1\right\}$ is closed, $\mu$ and $\hat{\mu}$ will be called slowly decreasing (or of regular growth) on the cone $\Gamma(A):=\{t a \mid a \in A, t \geq 0\}$ if the following holds: For each $\varepsilon>0$ there is $R>0$ such that for all $z \in \Gamma(A)$ with $|z| \geq R$ there is $w \in B(z, \varepsilon|z|)$ with $|\hat{\mu}(w)| \geq \exp (L(\bar{w})-\varepsilon|w|)$.

1.14. Remark. If $K=\{0\}$, i.e. if $P=\hat{\mu}$ is a nonzero entire function of at most order one and zero type, then $P$ is slowly decreasing on $C^{N}$ (see Martineau [15], Lemme 15).

The role of the strict convexity at $\partial_{r} \omega$ is shown by the following two results. The next one is a slight extension of a part of Maltsev [13], Cor. 1.

1.15. Proposition. If $Q \subset \mathrm{C}^{N}$ is strictly convex at $\partial_{r} \omega$, each nonzero differential operator of infinite order $P(D): A(Q) \rightarrow A(Q)$ and even each convolution operator $T_{\mu}: A(Q+K) \rightarrow A(Q)$ of which the symbol $\hat{\mu}$ is slowly decreasing on $\mathrm{C}^{N}$ is surjective. 
Proof. Let $\Omega$ be open in $\mathrm{C}^{N}$ with $Q \subset \Omega$. We show that there is a convex domain $\Omega_{0}$ in $\mathrm{C}^{N}$ with $Q \subset \Omega_{0} \subset \Omega$. Without loss of generality we may assume that $Q$ has nonempty interior, since otherwise $Q$ is compact.

For each $z \in \partial Q$ we choose a supporting hyperplane $R_{z}$ of $\operatorname{cl} Q$ at $z$, and denote by $P_{z}$ the open half space with boundary $R_{z}$ and with int $Q \subset P_{z}$.

For each $n \in \mathrm{N}$ we put $d_{n}:=\operatorname{dist}\left(\omega_{n}, \partial \Omega\right)>0$. For each $z \in \omega_{n} \backslash \omega_{n-1}$ $\left(\omega_{0}:=\emptyset\right)$, we put $A_{z}:=P_{z}+d_{n} U$, where $U$ is the open unit ball of $\mathrm{C}^{N}$. For each $z \in \partial Q \backslash \omega$, we put $A_{z}:=P_{z}$. (By the assumption on $Q$, we know that $Q \subset A_{z}$ for all $z \in \partial Q$. But we need more.) Now put $\Omega_{1}:=\cap_{z \in \partial Q} A_{z}$ and $\Omega_{0}:=\operatorname{int} \Omega_{1}$. Then $\Omega_{0}$ is a convex domain in $\mathrm{C}^{N}$ with $\Omega_{0} \subset \Omega$. We will show that $Q \subset \Omega_{0}$.

Obviously int $Q \subset \Omega_{0}$. Let $z \in \omega_{n} \backslash \omega_{n-1}$ for some $n \in \mathrm{N}$. As in Martineau [14], Lemme 1 of the proof of Thme. 1.2 (replace "complex supporting hyperplane" by "real supporting hyperplane" etc.), there is $m>n$ such that $c_{m}:=\inf _{w \in \partial Q \backslash \omega_{m}} \operatorname{dist}\left(z, R_{w}\right)>0$ and thus $t_{m}:=\min \left\{d_{m}, c_{m}\right\}>0$ which implies $z+t_{m} U \subset \Omega_{0}$ and thus $z \in \Omega_{0}$.

Since $\hat{\mu}$ is slowly decreasing on $\mathrm{C}^{N}$ (as $P$ is, by Remark 1.14), the operator $T_{\mu}: A\left(\Omega_{0}+K\right) \rightarrow A\left(\Omega_{0}\right)$ is surjective (Martineau [15], Morzhakov [29], Krivosheev [10], see also [21]). This shows that $T_{\hat{\mu}}: A(Q+K) \rightarrow A(Q)$ is surjective, too.

1.16. Proposition. Let $Q \subset \mathrm{C}^{N}$ be C-strictly convex at $\partial_{r} \omega$ (this is always the case for $N=1)$. If each nonzero partial differential operator $P(D): A(Q) \rightarrow A(Q)$ is surjective, then $Q$ is strictly convex at $\partial_{r} \omega$.

Proof. Assume that $Q$ is not strictly convex at $\partial_{r} \omega$. Then there is a supporting hyperplane $R=\left\{z \in \mathrm{C}^{N} \mid \operatorname{Re}\langle z, a\rangle=H(a)\right\}$ of $\operatorname{cl} Q$ such that $R \cap Q$ contains a line segment $I$ which is not relatively compact in $\omega$. After a translation of $Q$ we may assume that $I$ passes through the origin. After a unitary transformation of $\mathrm{C}^{N}$ we may assume that $\mathrm{C} I=\mathrm{C} \times\{0\}$. Hence $Q \cap \mathrm{C} I=Q^{\prime} \times\{0\}$ with a convex, locally closed, noncompact set $Q^{\prime} \subset \mathrm{C}$. Since $Q$ is C-strictly convex at $\partial_{r} \omega, Q^{\prime}$ has no interior points in C, i.e. $Q^{\prime}$ is a noncompact line segment (and in particular not strictly convex at $\partial_{r} \omega^{\prime}$ ). By Korobeŭnik [5], Thm. 2, there is a nonzero differential operator $P(D): A\left(Q^{\prime}\right) \rightarrow A\left(Q^{\prime}\right)$ of infinite order and a function $g \in A\left(Q^{\prime}\right)$ such that the equation $P(D) f=g$ has no solution $f \in A\left(Q^{\prime}\right)$.

By Martineau [14], Lemme 3 of the proof of Thme. 1.2, because of the strict convexity at $\partial_{r} \omega, Q$ has a neighborhood basis of linearly convex open sets, i.e. of open sets of which the complement is a union of complex hyperplanes. Those sets are pseudoconvex (see Hörmander [3], Prop. 4.6.3). Thus we can extend $g \in A\left(Q^{\prime}\right)$ to $G \in A(Q)$, by Hörmander [3], Thm. 4.2.12. If we consider $P(D)$ as an operator on $A(Q)$, by the hypothesis, there is $F \in A(Q)$ 
with $P(D) F=G$ and hence $P(D) F\left|Q^{\prime}=G\right| Q^{\prime}=g$. Since $f:=F \mid Q^{\prime} \in A\left(Q^{\prime}\right)$, this is a contradiction.

\section{An abstract criterion for the existence of a solution operator}

2.1. Notation. For all $n, m \in \mathrm{N}$, let $L_{n, m}$ denote the Hilbert space of all $f \in L_{l o c}^{2}\left(\mathrm{C}^{N}\right)$ such that

$$
\|f\|_{n, m}:=\left(\int_{\mathrm{C}^{N}}|f(z)|^{2} \exp \left(-2 H_{n}(\bar{z})-2|z| / m\right) d \lambda(z)\right)^{1 / 2}<\infty .
$$

We consider the Fréchet spaces $L_{\bar{H}_{n}}^{2}:=\operatorname{proj}_{m \rightarrow \infty} L_{n, m}, n \in \mathrm{N}$, and the (LF)space

$$
L_{\bar{H}}^{2}:=\operatorname{ind}_{n \rightarrow \infty} L_{\bar{H}_{n}}^{2}
$$

By $L_{\bar{H}_{n}(0,1)}^{2}$ and $L_{\bar{H}_{(0,1)}}^{2}$, we denote the corresponding Fréchet space of all $\bar{\partial}$-closed $(0,1)$-forms with coefficients in $L_{\bar{H}_{n}}^{2}$ and $L_{\bar{H}}^{2}$, respectively. For an open set $\Omega \subset \mathrm{C}^{N}$, we consider the Frechet spaces $W_{\bar{H}_{n}}^{2}\left(\mathrm{C}^{N}, \Omega\right)$ of all $f \in L_{H_{n}}^{2}$ with

$$
\bar{\partial} f \in L_{\bar{H}_{n}(0,1)}^{2} \text { and } f \mid \Omega \in A(\Omega)
$$

endowed with the norms $\left(\|f\|_{n, m}^{2}+\|\bar{\partial} f\|_{n, m}^{2}\right)^{1 / 2}, m \in \mathrm{N}$. We put

$$
W_{\bar{H}}^{2}\left(\mathrm{C}^{N}, \Omega\right):=\operatorname{ind}_{n \rightarrow \infty} W_{\bar{H}_{n}}^{2}\left(\mathrm{C}^{N}, \Omega\right) .
$$

By the mean value property of analytic functions, we have $A_{\bar{H}}=$ $W_{\bar{H}}^{2}\left(\mathrm{C}^{N}, \mathrm{C}^{N}\right)$.

2.2. Proposition. Let $\Omega \subset \mathrm{C}^{N}$ be open and assume that for each $a \in \mathrm{C}^{N} \backslash \Omega$ there is a plurisubharmonic function $u_{a}$ on $\mathrm{C}^{N}$ with $u_{a}(a) \geq 0$ satisfying the following condition: $\forall n \exists n^{\prime} \forall m \exists k, C>0$ :

$$
u_{a}(z) \leq H_{n^{\prime}}(\bar{z})+|z| / m-H_{n}(\bar{a})-|a| / k+C \quad \text { for all } \quad z \in \mathrm{C}^{N}, a \in \mathrm{C}^{N} \backslash \Omega .
$$

Then there is a continuous linear projection $P: W_{\bar{H}}^{2}\left(\mathrm{C}^{N}, \Omega\right) \rightarrow A_{\bar{H}}=$ $W_{\bar{H}}^{2}\left(\mathrm{C}^{N}, \mathrm{C}^{N}\right)$.

Proof. As in [17], 1.8, the assertion follows from Langenbruch [11], Thm. 1.3 and Remark 1.11.

2.3. Notation. If $F$ is an entire function, we put $V(F):=\left\{z \in \mathrm{C}^{N}\right.$ $F(z)=0\}$. By $A_{F} \subset S$, we denote the set of all $a \in S$ such that 
$a=\lim _{j \rightarrow \infty} a_{j} /\left|a_{j}\right|$ for some sequence $\left(a_{j}\right)_{j \in \mathrm{N}}$ in $V(F)$ with $\lim _{j \rightarrow \infty}\left|a_{j}\right|=\infty$. Its tangent cone at infinity is defined by

$$
V_{\infty}(F):=\Gamma\left(A_{F}\right):=\left\{t a \mid t \geq 0, a \in A_{F}\right\} .
$$

We note that $\operatorname{dist}\left(a, V_{\infty}(F)\right)=o(|a|)$ as $a \in V(F)$ tends to infinity.

From [17], Lemma 2.2, we recall:

2.4. Lemma. Let $\mu \in A(K)^{\prime}$ be slowly decreasing on $V_{\infty}(\hat{\mu})$. Then there is a locally bounded function $r: \mathrm{C}^{N} \rightarrow[1, \infty[$ with $r(z)=o(|z|)$ for $z \rightarrow \infty$ and such that for each $\varepsilon>0$ there is $R>0$ with the following property: Whenever $z \in \mathrm{C}^{N}$ and $|z| \geq R$ satisfies $U(z, r(z)) \cap V(\hat{\mu}) \neq \emptyset$, then there is $w \in U(z,(1+\varepsilon) r(z))$ with $|\hat{\mu}(w)| \geq \exp (L(\bar{w})-\varepsilon|w|)$. We put

$$
r^{\prime}(z):=\sup \left\{|z-w|+2 r(w)\left|w \in \mathrm{C}^{N},\right| z-w \mid \leq r(z)+r(w)\right\}, \quad z \in \mathrm{C}^{N} .
$$

Then $2 r \leq r^{\prime}$ and $r^{\prime}(z)=o(|z|)$ for $|z| \rightarrow \infty$. If $U(z, r(z)) \cap U(w, r(w)) \neq \emptyset$, then also $U(w, 2 r(w)) \subset U\left(z, r^{\prime}(z)\right)$.

2.5. Auxiliary spaces. Let $\mu \in A(K)^{\prime}$ be slowly decreasing on $V_{\infty}(\hat{\mu})$. For each open set $\Omega \subset \mathrm{C}^{N}$, let $A^{2}(\Omega)$ be the Hilbert space of all square integrable functions in $A(\Omega)$. Let $I(\Omega)$ be its closed subspace $I(\Omega)=(\hat{\mu} \cdot A(\Omega)) \cap$ $A^{2}(\Omega)$. We put $E_{\Omega}:=A^{2}(\Omega) / I(\Omega)$ and for $x_{\Omega} \in E(\Omega)$

$$
\left|x_{\Omega}\right|_{\Omega}:=\inf _{\xi \in x_{\Omega}}|\xi|_{2}=\inf _{\xi \in x_{\Omega}}\left(\int_{\Omega}|\xi|^{2} d \lambda\right)^{1 / 2} .
$$

We choose $r^{\prime}: C^{N} \rightarrow\left[1, \infty\left[\right.\right.$ according to Lemma 2.4 , and set $\tilde{r}:=16 r^{\prime}$. For each $z \in \mathrm{C}^{N}$, we write $\Omega(z):=U(z, \tilde{r}(z))$.

We put $A_{\bar{H}+\bar{L}}(\hat{\mu}):=\operatorname{ind}_{n \rightarrow \infty} A_{\bar{H}_{n}+\bar{L}}^{0}(\hat{\mu})$, where for each $n \in \mathrm{N}, A_{\bar{H}_{n}+\bar{L}}^{0}(\hat{\mu})$ is the space of all $x=\left(f_{\Omega(z)}+I(\Omega(z))\right)_{z \in \mathrm{C}^{N}} \in \prod_{z \in \mathrm{C}^{N}} E_{\Omega(z)}$ with

$$
f_{\Omega(z)}-f_{\Omega(w)} \in I(\Omega(z) \cap \Omega(w)) \text { whenever } \Omega(z) \cap \Omega(w) \neq \emptyset
$$

and

$$
\|x\|_{n, m}:=\sup _{z \in \mathrm{C}^{N}}\left|x_{\Omega(z)}\right|_{\Omega(z)} \exp \left(-H_{n}(\bar{z})-|z| / m-L(\bar{z})\right)<\infty
$$

for all $m \in \mathrm{N}$.

2.6. Proposition. Let $\mu \in A(K)^{\prime}$ be slowly decreasing on $V_{\infty}(\hat{\mu})$ such that $\left(\hat{\mu} \cdot A\left(\mathrm{C}^{N}\right)\right) \cap A_{\bar{H}+\bar{L}}=\hat{\mu} \cdot A_{\bar{H}}$. Then the linear mapping

$$
\bar{\rho}: A_{\bar{H}+\bar{L}} /\left(\hat{\mu} \cdot A_{\bar{H}}\right) \rightarrow A_{\bar{H}+\bar{L}}(\hat{\mu}), \quad \bar{\rho}\left(f+\hat{\mu} \cdot A_{\bar{H}}\right):=(f \mid \Omega(z)+I(\Omega(z)))_{z \in \mathrm{C}^{N}}
$$

is an isomorphism of (LF)-spaces. Moreover: $\forall n, m \exists k, C>0$ : 
(a) $\left\|\bar{\rho}\left(f+\left(\hat{\mu} \cdot A_{\bar{H}}\right) \cap A_{\bar{H}_{n}+\bar{L}}^{0}\right)\right\|_{n, m} \leq C\left\|f+\left(\hat{\mu} \cdot A_{\bar{H}}\right) \cap A_{\bar{H}_{n}+\bar{L}}^{0}\right\|_{n, k} \quad$ for $\quad$ all $f \in A_{\bar{H}_{n}+\bar{L}}^{0}$

(b) $\left\|\bar{\rho}^{-1}(x)\right\|_{n, m} \leq C\|x\|_{n, k}$ for all $x \in A_{\bar{H}_{n}+\bar{L}}^{0}(\hat{\mu})$.

Proof. By [17], Prop. 2.4, for each $n \in \mathrm{N}$,

$$
\rho: A_{\bar{H}_{n}+\bar{L}}^{0} \rightarrow A_{\bar{H}_{n}+\bar{L}}^{0}(\hat{\mu}), \quad \rho(f):=(f \mid \Omega(z)+I(\Omega(z)))_{z \in \mathrm{C}^{N}},
$$

is a well defined surjective continuous linear map. This shows that

$$
\rho: A_{\bar{H}+\bar{L}} \rightarrow A_{\bar{H}+\bar{L}}(\hat{\mu})
$$

has the same properties. The postulated estimates follow from the corresponding ones in [17], Prop. 2.4. The kernel of $\rho$ is $\left(\hat{\mu} \cdot A\left(\mathrm{C}^{N}\right)\right) \cap A_{\bar{H}+\bar{L}}=\hat{\mu} \cdot A_{\bar{H}}$. Hence by the definition of the inductive limit topology on $A_{\bar{H}+\bar{L}} /\left(\hat{\mu} \cdot A_{\bar{H}}\right), \bar{\rho}$ has the announced properties.

2.7. Proposition. Let $\mu$ be as in Proposition 2.6. If for each $a \in A_{\hat{\mu}}$ there is a plurisubharmonic function $u_{a}$ on $\mathrm{C}^{N}$ with $u_{a}(a) \geq 0$ satisfying the condition: $\forall n \exists n^{\prime} \forall m \exists k$ :

$$
u_{a}(z) \leq H_{n^{\prime}}(\bar{z})+|z| / m-H_{n}(\bar{a})-1 / k \quad \text { for all } z \in \mathrm{C}^{N}, a \in A_{\hat{\mu}},
$$

then the quotient map $A_{\bar{H}+\bar{L}} \rightarrow A_{\bar{H}+\bar{L}} /\left(\hat{\mu} \cdot A_{\bar{H}}\right)$ has a continuous linear right inverse.

Proof. As in [17], Cor. 2.5, but now applying Proposition 2.2.

2.8. Roposition. Let $\mu \in A(K)^{\prime}$ be slowly decreasing on $\mathrm{C}^{N}$ and such that $T_{\mu}: A(Q+K) \rightarrow A(Q)$ is surjective. Then $T_{\mu}: A(Q+K) \rightarrow A(Q)$ admits a solution operator if the following holds: For each $a \in A_{\hat{\mu}}$ there is a plurisubharmonic function $u_{a}$ on $\mathrm{C}^{N}$ with $u_{a}(a) \geq 0$ such that: $\forall n \exists n^{\prime} \forall m \exists k$ with

$$
u_{a}(z) \leq H_{n^{\prime}}(\bar{z})+|z| / m-H_{n}(\bar{a})-1 / k \quad \text { for all } z \in \mathrm{C}^{N}, a \in A_{\hat{\mu}} .
$$

Proof. By Proposition 2.7, and Lemmas 1.10 and 1.12.

\section{Evaluation of the criterion}

In this chapter we shall evaluate the abstract condition of Proposition 2.8.

3.1. Definition. We recall that $S:=\left\{a \in \mathrm{C}^{N}|| a \mid=1\right\}$ and $\Gamma(B):=$ $\{t x \mid t \geq 0, x \in B\}$, whenever $B \subset \mathrm{C}^{N}$. We will say that $A \subset S$ satisfies the condition $U(A, Q)$ if for each $a \in A$ there is a plurisubharmonic function $u_{a}$ on $\mathrm{C}^{N}$ with $u_{a}(a) \geq 0$ such that: $\forall n \exists n^{\prime} \forall m \exists k$ with

$$
u_{a}(z) \leq H_{n^{\prime}}(\bar{z})+|z| / m-H_{n}(\bar{a})-1 / k \quad \text { for all } z \in \mathrm{C}^{N}, a \in A .
$$


Recall that $Q \subset \mathrm{C}^{N}$ is assumed to be nonpluripolar.

We recall some definitions from [27] and [25]:

3.2. Definition. If $Q \subset \mathrm{C}^{N}$ is bounded and $c>0$, let $v_{H, c}^{0}$ be the largest plurisubharmonic function on $\mathrm{C}^{N}$ bounded by $H$ and with $v_{H, c}^{0}(z) \leq$ $c \log |z|+O(1)$ as $|z| \rightarrow 0$. A function $C_{H}^{0}: S \rightarrow[0, \infty[$ is defined by

$$
\left\{z \in \mathrm{C}^{N} \mid v_{H, c}^{0}(z)=H(z)\right\}=\left\{\lambda a \mid a \in S, 1 / C_{H}^{0}(a) \leq \lambda<\infty\right\} .
$$

If $Q$ is bounded and with int $Q \neq \emptyset$, with $0 \in \operatorname{int} Q$, and if $C>0$, let $v_{H, C}^{\infty}$ be the largest plurisubharmonic function on $\mathrm{C}^{N}$ bounded by $H$ and with $v_{H, C}^{\infty}(z) \leq C \log |z|+O(1)$ as $|z| \rightarrow \infty$. A function $\left.\left.C_{H}^{\infty}: S \rightarrow\right] 0, \infty\right]$ is defined by

$$
\left\{z \in \mathrm{C}^{N} \mid v_{H, C}^{\infty}(z)=H(z)\right\}=\left\{\lambda a \mid a \in S, 0 \leq \lambda \leq 1 / C_{H}^{\infty}(a)\right\} .
$$

3.3. Notation. Let $Q \subset \mathrm{C}^{N}$. For $\gamma \subset Q$ and $A \subset S$, we define

$$
S_{\gamma}:=\{a \in S \mid \operatorname{Re}\langle w, a\rangle=H(a) \text { for some } w \in \gamma\}
$$

and

$$
F_{A}:=\{w \in Q \mid \operatorname{Re}\langle w, a\rangle=H(a) \text { for some } a \in A\} .
$$

We will write $S_{o}:=S \backslash S_{Q}$. We note that $a \in S_{o}$ if and only if $H_{n}(a)<H(a)$ for all $n \in \mathrm{N}$. If int $Q \neq \emptyset$, obviously $S_{\omega}=S_{Q}$.

3.4. Lemma. Let $Q \subset \mathrm{C}^{N}$ be bounded, with nonempty interior, strictly convex at $\partial_{r} \omega$. If $A \subset S_{\omega}$ is compact then $F_{A} \subset \omega$ is compact.

Proof. If $w_{n}$ is a sequence in $F_{A}$, there is a sequence $a_{n}$ in $A$ with $\operatorname{Re}\left\langle w_{n}, a_{n}\right\rangle=H\left(a_{n}\right), n \in \mathrm{N}$. By the compactness of $A$ and $\operatorname{cl} \omega$, we may assume that the sequences $a_{n}$ and $w_{n}$ converge to $a \in A$ and $w \in \operatorname{cl} \omega$, respectively. Hence $\operatorname{Re}\langle w, a\rangle=H(a)=\operatorname{Re}\langle z, a\rangle$ for some $z \in \omega$. Thus $w \in \omega$ and hence in $F_{A}$, since otherwise $F_{\{a\}}$ would not be compact violating the assumption of strict convexity at $\partial_{r} \omega$.

3.5. Lemma. Let $Q \subset \mathrm{C}^{N}$ be bounded with nonempty interior. Then (i) $\Leftrightarrow$ (ii) $\Rightarrow$ (iii):

(i) $Q$ is strictly convex at $\partial_{r} \omega$.

(ii) For each compact $\gamma_{1} \subset \omega$ there is a compact $\gamma_{2}$ such that $\gamma_{1} \subset \gamma_{2} \subset \omega$ and $F_{\{a\}}$ does not intersect $\gamma_{1}$ and $\omega \backslash \gamma_{2}$ for some $a \in S_{\omega}$, i.e. such that there is no line segment in $\partial Q$ that meets $\gamma_{1}$ and $\omega \backslash \gamma_{2}$.

(iii) $S_{\omega}$ is open in $S$ and $\left(S_{\omega_{n}}\right)_{n \in \mathrm{N}}$ is a compact exhaustion of $S_{\omega}$.

Proof. (i) $\Rightarrow$ (ii): Put $A:=S_{\gamma_{1}}$. By Lemma 3.4, $F_{A} \subset \omega$ is compact. 
Choose $\gamma_{2} \subset \omega$ with $F_{A} \cap\left(\omega \backslash \gamma_{2}\right)=\emptyset$. If $F_{\{a\}} \cap \gamma_{1} \neq \emptyset$ then $a \in A$ and thus $F_{\{a\}} \cap\left(\omega \backslash \gamma_{2}\right)=\emptyset . \quad$ (ii) $\Rightarrow$ (i) is trivial.

(i) $\Rightarrow$ (iii): By Lemma 3.4, $\left(S_{\omega_{n}}\right)_{n \in \mathrm{N}}$ is a compact exhaustion of $S_{\omega}$, since $\left(\omega_{n}\right)_{n \in \mathrm{N}}$ is a compact exhaustion for $\omega$. It easily follows from the definition of strict convexity at $\partial_{r} \omega$ that $S_{\omega}$ is open.

3.6. Proposition. Let $Q \subset \mathrm{C}^{N}$ be bounded with 0 in its nonempty interior, and which is strictly convex at $\partial_{r} \omega$. Then for a given compact set $A \subset S$, the condition $U(A, Q)$ is fulfilled if and only if $C_{H}^{\infty}$ is bounded on a neighborhood of $A_{o}:=\bar{A} \cap S_{o}$ in $\bar{A}$ and $1 / C_{H}^{0}$ is bounded on each compact subset of $A_{\omega}:=\bar{A} \cap S_{\omega}$.

Proof. Note that by Lemma 3.5, $S_{\omega}$ is open in $S$ and $S_{o}$ is compact.

" $\Rightarrow$ ": We put $v:=\left(\sup _{a \in \bar{A}}\left(\bar{u}_{\bar{a}}+H(a)\right)\right)^{*}$. Then $v$ is plurisubharmonic on $\mathrm{C}^{N}$ with $v \geq H$ on $\bar{A}$ and $\forall n \exists n^{\prime} \forall m \exists k$ such that

$$
v \leq H_{n^{\prime}}+|\cdot| / m+1 / n-1 / k .
$$

Hence $v \leq H$ and in particular $v=H$ on $\bar{A}$. Choose $n^{\prime}$ for $n=1$ according to (1). Since $H_{n^{\prime}}(a)<H(a)$ for all $a \in S_{o}$, by the compactness of $S_{o}$ there is some $m$ with $H_{n^{\prime}}(a)+|a| 2 / m<H(a)$ for $a \in S_{o}$, and hence there is some compact $S_{1} \subset S_{\omega}$ such that $H_{n^{\prime}}+|\cdot| 2 / m \leq H$ holds on the cone $\Gamma\left(S \backslash S_{1}\right)$. If $k$ is chosen according to (1), we obtain

(2) $\quad v(z) \leq H_{n^{\prime}}(z)+|z| / m+1 / 1-1 / k \leq H(z)-|z| / m+1, \quad z \in \Gamma\left(S \backslash S_{1}\right)$.

For the compact set $\gamma_{1}:=F_{S_{1}} \subset \omega$, we have $S_{1} \subset S_{\gamma_{1}} \subset S_{\omega}$. According to Lemma 3.5 (ii) we can choose $\gamma_{1} \subset \gamma_{2}$ open and relatively compact in $\omega$ such that there is no $a \in S_{\omega}$ such that $F_{\{a\}}$ intersects $\gamma_{1}$ and $\omega \backslash \gamma_{2}$. As in [25], Prop. 2.2, we consider

$$
L(z):=\sup _{w \in \partial Q \backslash \gamma_{2}} \operatorname{Re}\langle w, z\rangle=\max _{w \in \partial Q \backslash \gamma_{2}} \operatorname{Re}\langle w, z\rangle, \quad z \in \mathrm{C}^{N} .
$$

Obviously $L=H$ on $S \backslash S_{\gamma_{2}}$. If $L(a)=H(a)$, then $\operatorname{Re}\langle w, a\rangle=H(a)$ for some $w \in \partial Q \backslash \gamma_{2}$, hence $a \notin S_{\gamma_{1}}$, since otherwise we would get a contradiction with 3.5 (ii). This proves $L<H$ on $S_{\gamma_{1}}$.

Since $S \backslash S_{\gamma_{2}} \subset S \backslash S_{1}$, by (2) (for large $|z|$ ), there is some $R>1$ such that the plurisubharmonic function $\tilde{v}:=v / 2+L / 2$ is strictly smaller than $H$ outside $[0, R] \cdot\left(S \backslash S_{\gamma_{2}}\right)$. Moreover, $\tilde{v} \leq H$ on $\mathrm{C}^{N}$, and $\tilde{v}=H$ on $\bar{A} \cap\left(S \backslash S_{\gamma_{2}}\right)$. By [25], 2.1 Lemma, this implies that $C_{H}^{\infty}$ is bounded on $\bar{A} \cap\left(S \backslash S_{\gamma_{2}}\right)$.

Now let $\kappa$ be a compact subset of $A_{\omega}$. By Lemma 3.5 (iii), there is some $n$ with $\kappa \subset S_{\omega_{n}}$. The plurisubharmonic function $v:=\left(\sup _{a \in \bar{\kappa}}\left(\bar{u}_{\bar{a}}+H(a)\right)\right)^{*}$ satisfies $v \geq H$ on $\bar{\kappa}$. Since $H=H_{n}$ on $S_{\omega_{n}}$, there is $n^{\prime}$ such that for all $m$ there exists $k$ with 


$$
v \leq H_{n^{\prime}}+|\cdot| / m+0-1 / k
$$

Hence $v \leq H_{n^{\prime}} \leq H$, in particular $v=H$ on $\bar{\kappa}$. Since $v(0) \leq-1 / k<0$ (for $m=1$ ), it follows from [27], 2.14, that $1 / C_{H}^{0}$ (and even $1 / C_{H_{n^{\prime}}}^{0}$ ) is bounded on $\bar{\kappa}$.

" $\Leftarrow$ ": By the hypotheses, there are a neighborhood $\tilde{A}$ of $A_{o}$ in $\bar{A}$ and some (large) $C>0$ such that $v_{H, C}^{\infty}=H$ on $\tilde{A}$. Let $n \in \mathrm{N}$. Since $H_{n}<H$ on $A_{o}$, there is an open neighborhood $A_{n}$ of $A_{o}$ in $\bar{A}$ with $H_{n}<H$ on $\operatorname{cl} A_{n}$. We may assume $A_{1} \subset \tilde{A}$ and $A_{n} \subset A_{n-1}$. By the hypotheses, there is some (small) $c_{n}>0$ with $v_{H, c_{n}}^{0}=H$ on $\bar{A} \backslash A_{n}$.

Fix $n$ and put $\kappa:=\bar{A} \backslash A_{n}$. We claim that - essentially - we may replace $v_{H, c_{n}}^{0}$ by $v_{H_{n^{\prime}}, c_{n}^{\prime}}^{0}$, for some $n^{\prime}$ and some $c_{n}^{\prime}$. The following suffices: By Lemma 3.5 , the set $F_{\kappa} \subset \omega$ is compact and thus contained in $Q_{\tilde{n}}$ for some $\tilde{n}$. We claim that there is $n^{\prime} \geq \tilde{n}$ with

$$
\frac{1}{2} H+\frac{1}{2} H_{\tilde{n}} \leq H_{n^{\prime}}
$$

If $w \in \operatorname{cl} Q$ and $z \in Q_{\tilde{n}}$, we have either $w / 2+z / 2 \in \operatorname{intcl} Q$ and hence in $Q$, or $z, w \in \partial \operatorname{cl} Q$, i.e. $z \in \omega_{\tilde{n}}$, and $[z, w] \subset \partial Q$. Since $Q$ is strictly convex at $\partial_{r} \omega$, we obtain $[z, w] \subset \omega$, in particular $z / 2+w / 2 \in Q$. We may of course assume that $(n+1)^{\prime}>n^{\prime}$. Define

$$
v_{n}:=\frac{1}{2} v_{H, c_{n}}^{0}+\frac{1}{2} H_{\tilde{n}} .
$$

$v_{n}$ is plurisubharmonic, $v_{n}=H$ on $\kappa, v_{n}(0)<0$, and $v_{n} \leq H_{n^{\prime}}$.

For $a \in A_{o}$, we put $v_{a}:=v_{H, C}^{\infty}$. If $a \in \bar{A} \backslash A_{1}$ we set $v_{a}:=v_{1}$. For each $n>1$ and $a \in A_{n-1} \backslash A_{n}$, we put $v_{a}:=\frac{1}{2} v_{H, C}^{\infty}+\frac{1}{2} v_{n}$. Then $v_{a}(a)=H(a), a \in \overline{A_{\hat{\mu}}}$, in all three cases. In the last case this is true because $v_{H, C}^{\infty}=H$ on $A_{1}$.

Now, let $n$ be given. Since $H_{n}<H$ on $\operatorname{cl} A_{n}$, we may assume that $n^{\prime}$ is in addition chosen such that

$$
v_{H, C}^{\infty} \leq H_{n^{\prime}}+\min _{a \in \operatorname{cl} A_{n}}\left(H(a)-H_{n}(a)\right) / 2 .
$$

If $m$ is given, by [17], Lemma 2.9 (applied with $H_{j^{\prime}}$ instead of $H$ ), we can choose $k$ so large that

$$
v_{j} \leq H_{j^{\prime}}+|\cdot| / m-1 / k \quad \text { for all } j=1, \ldots, n,
$$

and $1 / k \leq \min _{a \in \mathrm{cl} A_{n}}\left(H(a)-H_{n}(a)\right) / 2$. If $a \in A_{n}$ we obtain $v_{H, C}^{\infty} \leq H_{n^{\prime}}+\min _{a \in \mathrm{cl} A_{n}}\left(H(a)-H_{n}(a)\right)-1 / k \leq H_{n^{\prime}}+|\cdot| / m+H(a)-H_{n}(a)-1 / k$.

This is the desired estimate of $v_{a}$ if $a \in A_{o}$. For $a \in A_{n} \backslash A_{o}$ this estimate follows from

$$
v_{a} \leq \frac{1}{2}\left(H_{n^{\prime}}+|\cdot| / m+H(a)-H_{n}(a)-1 / k\right)+\frac{1}{2} H,
$$


since - by the strict convexity of $Q$ at $\partial_{r} \omega$ - we have $\frac{1}{2} H_{n^{\prime}}+\frac{1}{2} H \leq H_{n^{\prime \prime}}$ for some $n^{\prime \prime}$.

By the choice of $k$, for all $a \in S$ and $j=1, \ldots, n$

$$
v_{j} \leq H_{n^{\prime}}+|\cdot| / m+H(a)-H_{n}(a)-1 / k
$$

holds, which implies in particular the desired estimate of $v_{a}$ if $a \in \bar{A} \backslash A_{1}$. Moreover, we get for $a \in A_{1} \backslash A_{n}$ that

$$
v_{a} \leq \frac{1}{2} H+\frac{1}{2}\left(H_{n^{\prime}}+|\cdot| / m+H(a)-H_{n}(a)-1 / k\right) .
$$

Again the strict convexity of $Q$ at $\partial_{r} \omega$ implies the desired estimate for $v_{a}$ in this case.

Finally, we define

$$
u_{a}(z):=v_{\bar{a}}(\bar{z})-H(\bar{a}), \quad a \in A .
$$

3.7. Corollary. The assertion of Proposition 3.6 also holds if we replace in the condition $U(A, Q)$ the numbers $H_{n}(\bar{a})+1 / k$ by numbers $H_{n, k}(\bar{a}) \leq$ $H_{n}(\bar{a})+1 / k, a \in A$, which are increasing in $n$ and decreasing in $k$ and have the following properties:

(i) $\lim _{n \rightarrow \infty} \inf _{k \in \mathrm{N}} H_{n, k}(\bar{a})=H(\bar{a})$ uniformly on $A$.

(ii) For each $\kappa \subset S_{\omega} \cap \bar{A}$ compact and each $n$ there is some $m$ such that for each $k$ there is $l$ with

$$
H_{n}(a)+1 / l \leq H_{m, k}(a), \quad a \in \kappa .
$$

Proof. In the proof of 3.6 " $\Rightarrow$ ", we apply (i) to get (1) in the proof of the bounds of $C_{H}^{\infty}$, while (ii) is applied in the proof of the bounds of $C_{H}^{0}$. " $\Leftarrow$ " follows immediately from Proposition 3.6.

3.8. Lemma. Let $Q \subset \mathrm{C}^{N}$ be bounded. For a $\in S$ let $L_{a}+a$ be a complex supporting hyperplane for $\left\{z \in \mathrm{C}^{N} \mid H(z) \leq H(a)\right\}$ at a. For $n, k \in \mathrm{N}$ define

$$
H_{n, k}(a):=\min _{z \in L_{a}+a}\left(H_{n}(z)+|z| / k\right) .
$$

Let $\kappa=\{a\} \subset S$ only have a single element or if $Q$ has nonempty interior and is strictly convex at $\partial_{r} \omega$ let $\kappa$ be a compact subset of $S_{\omega}$. Then for each $n$ there is some $m$ such that for each $k$ there is $l$ with

$$
H_{n, l}(a) \leq H_{n}(a)+|a| / l \leq H_{m, k}(a), \quad a \in \kappa .
$$

Furthermore, $\lim _{m \rightarrow \infty} \inf _{k \in \mathrm{N}} H_{m, k}(a)=H(a)$ uniformly on $S$.

Proof. We note that after a translation of $Q$ we may assume that $0 \in \operatorname{int}_{r} Q$. If $\kappa \subset S_{\omega}$ is compact, by Lemma 3.5 , there is $n_{0} \in \mathrm{N}$ such that $F_{\kappa} \subset Q_{n_{0}}$, and we conclude that $L_{a}+a \subset\left\{z \in \mathrm{C}^{N} \mid \operatorname{Re}\left\langle w_{a}, z\right\rangle=H(a)\right\}$ with 
$w_{a} \in Q_{n_{0}}$. This shows that $L_{a}+a$ is also a complex supporting hyperplane for the sets $\left\{z \in \mathrm{C}^{N} \mid H_{m}(z) \leq H_{m}(a)\right\}$ for all $m \geq n_{0}$. Hence for all $m \geq n_{0}$

$$
\min _{z \in L_{a}+a}\left(H_{m}(z)+H(z) / k\right) \geq \min _{z \in L_{a}+a} H_{m}(z)+\min _{z \in L_{a}+a} H(z) / k=H_{m}(a)+H(a) / k,
$$

which proves the first part of the assertion also for $\kappa=\{a\}$ if $a \in S_{\omega}$ in the general case.

For the proof of the second part, we may assume that $Q_{m}=\operatorname{cv}\left(K_{m}, \omega_{m}\right)$ as in Lemma 1.2, where $K_{m}:=(1-1 / m) \operatorname{cl} Q$. Thus we get the lower bound

$$
H_{m, k}(a) \geq(1-1 / m) H(a)+H(a) / k \geq(1-1 / m) H(a), \quad a \in S,
$$

for all $m$ and $k$. This also proves the first part for $\kappa=\{a\}$ if $a \in S_{o}$.

By Proposition 1.15, the strict convexity of $Q$ at $\partial_{r} \omega$ is sufficient for all nonzero $P(D): A(Q) \rightarrow A(Q)$ to be surjective. By Propositions 1.16 this condition is also necessary in the case $N=1$.

3.9. TheOREM. Let $Q \subset \mathrm{C}^{N}$ be strictly convex at $\partial_{r} \omega$, bounded, with 0 in its nonempty interior. Then each nonzero partial differential operator $P(D)$ : $A(Q) \rightarrow A(Q)$ has a continuous linear right inverse if and only if $C_{H}^{\infty}$ is bounded on a neighborhood of $S_{o}$ in $S$, and $1 / C_{H}^{0}$ is bounded on each compact subset of $S_{\omega}$.

Proof. The sufficiency: By Proposition 3.6, the condition $U(S, Q)$ is fulfilled. By Proposition 2.8 together with Definition 1.11 and Remark 1.14, each $P(D): A(Q) \rightarrow A(Q)$ admits a continuous linear right inverse.

The necessity: Choose a dense sequence $\left(a_{j}\right)_{j \in \mathrm{N}}$ in $S$. Fix $j$. Let $L_{a_{j}}+a_{j}$ be a complex supporting hyperplane for $\left\{z \in \mathrm{C}^{N} \mid H(\bar{z}) \leq H\left(\bar{a}_{j}\right)\right\}$ at $a_{j}$. We choose rapidly increasing sequences $\left(\lambda_{j, l}\right)_{l \in \mathrm{N}}$ of positive reals, such that the canonical product $P_{j} \in A\left(\mathrm{C}^{N}\right)$ with $V\left(P_{j}\right)=\cup_{l \in \mathrm{N}} L_{a_{j}}+\lambda_{j, l} a_{j}$ converges, and such that even the product $P=\prod_{j \in \mathrm{N}} P_{j}$ converges and defines a function in $A_{\{0\}}^{0}$ (see [22], Lemma 2.5).

By the definition of $L_{a_{j}}+a_{j}$ we have $H\left(\bar{a}_{j}\right)=\min _{z \in L_{a_{j}}+a_{j}} H(\bar{z})$. For $n, k$ and $a \in S$ we define

$$
H_{n, k}(\bar{a}):=\min _{z \in L_{a}+a}\left(H_{n}(\bar{z})+|z| / k\right) .
$$

If $P(D)$ has a right inverse, $M_{P}$ has a left inverse $L$, hence each $M_{P_{j}}$ has the left inverse $L \circ M_{P / P_{j}}$, and the corresponding quotient map $\rho_{j}: A_{\bar{H}} \rightarrow A_{\bar{H}}\left(P_{j}\right)$ in the proof of Proposition 2.6 has the right inverse

$$
R_{j}\left(\rho_{j}(f)\right):=f-M_{P_{j}} \circ L \circ M_{P / P_{j}}(f)=f-P_{j} L\left(f P / P_{j}\right) .
$$

As in [22], Lemma 2.5, it follows that the condition $U(S, Q)$ is fulfilled with 
$H_{n}(\bar{a})-1 / k$ replaced by $H_{n, k}(\bar{a})$. Now because of Lemma 3.8, by Corollary 3.7, the functions $C_{H}^{\infty}$ and $C_{H}^{0}$ have the postulated bounds.

The following propositions show that in fact it is no restriction to consider in Theorem 3.9 only bounded $Q$ with nonempty interior.

3.10. Proposition. If $Q \subset \mathrm{C}^{N}$ is strictly convex at $\partial_{r} \omega$, the following are equivalent:

(i) Each nonzero $P(D): A(Q) \rightarrow A(Q)$ admits a continuous linear right inverse.

(ii) After a unitary transformation of $\mathrm{C}^{N}, Q$ equals the Cartesian product $Q^{\prime} \times \mathrm{C}^{N^{\prime \prime}}$, where $Q^{\prime} \subset \mathrm{C}^{N^{\prime}}$ is bounded, with nonempty interior, such that $P^{\prime}(D): A\left(Q^{\prime}\right) \rightarrow A\left(Q^{\prime}\right)$ admits a continuous linear right inverse for each nonzero $P^{\prime}(z)=\sum_{\alpha \in \mathrm{N}_{0}^{N^{\prime}}} a_{\alpha} z^{\alpha}$ of $N^{\prime}$ variables, and such that either $Q=Q^{\prime}$ or $Q$ is open.

Proof. (i) $\Rightarrow$ (ii): In any case, after a unitary transformation we have the representation with some $Q^{\prime} \subset \mathrm{C}^{N^{\prime}}$ such that the set $\left\{z \in \mathrm{C}^{N^{\prime}} \mid H^{\prime}(z)<\infty\right\}$ is not pluripolar in $\mathrm{C}^{N^{\prime}}$ (see [22], Thm. 2.7). Obviously, $Q^{\prime}$ is again locally closed and convex. Since $Q^{\prime} \times \mathrm{C}^{N^{\prime \prime}}$ is strictly convex at $\partial_{r} \omega, N^{\prime \prime}=0$ unless the $Q^{\prime} \subset \mathrm{C}^{N^{\prime}}$ is open. In the second case, the assertion follows from [22], Thm. 2.7. In the first case, we have to show that $Q=Q^{\prime}$ is bounded with nonempty interior. First we show that $Q$ is bounded: Assume that $Q$ is not bounded. Then there is some $a \in S$ with $\mathrm{R}_{+} a \subset \bar{Q}$ (we may assume that $0 \in Q$ ). Furthermore we may assume that the compact exhaustion of $Q$ is chosen such that the hyperplane $\left\{z \mid \operatorname{Re}\langle z, a\rangle=H_{n}(a)\right\}$ touches $\bar{Q}_{n}$ in some point of $\Gamma(a)$. Let $L_{a}+a$ denote the complex hyperplane which is contained in this real one. We now proceed as in [22], Thm. 2.7, and get a partial differential operator $P(D)$ of infinite order, such that the zeros of $P$ are positive multiples of $L_{a}+a$. Since $P(D)$ admits a continuous linear right inverse on $A(Q)$, as in [22], Lemma 2.5 (applying the proof of Proposition 2.6), i.e. as in Theorem 3.9, we see that condition $U(\{a\}, Q)$ is fulfilled. Hence for all $n$

$$
u_{a}(z) \leq H(\bar{z})-H_{n}(\bar{a}), \quad z \in \mathrm{C}^{N} .
$$

Since $\lim _{n \rightarrow \infty} H_{n}(\bar{a})=\infty$, this implies that the nonzero plurisubharmonic function $u_{a}$ equals $-\infty$ on $\{z \mid H(\bar{z})<\infty\}$. This contradicts the definition of $Q^{\prime}$.

We show that $Q$ has nonempty interior: Assume that the interior is empty. Then there is $b \in S$ such that $Q \subset\left\{z \in \mathrm{C}^{N} \mid \operatorname{Re}\langle z, b\rangle=0\right\}$. Put $a:=i \bar{b}$. Choose $L_{a}+a$ to be the complex hyperplane which contained in $\{z \mid \operatorname{Re}\langle z, a\rangle=H(\bar{a})\}$. We choose $P(D)$ as above. Since $P(D)$ admits a continuous linear right inverse, we see as above - but now applying Lemma 3.8 
- that condition $U(\{a\}, Q)$ is fulfilled. As in the proof of Proposition 3.6 we see that $v_{H}^{0}(\bar{a})=H(\bar{a})$. On the other side $\zeta \mapsto H(\zeta \bar{a})$ vanishes for $\pm i$ which implies that the positively homogeneous function $H(\zeta a)$ is harmonic for $\operatorname{Re} \zeta>0$. Since $v_{H}^{0}(0)<0$ this yields a contradiction, applying the maximum principle to the subharmonic function $\zeta \mapsto v_{H}^{0}(\zeta \bar{a})-H(\zeta \bar{a})$.

(ii) $\Rightarrow$ (i): If $Q$ is open, the assertion has been proved in [22], Thm. 2.7. If $Q=Q^{\prime}$ there is nothing to prove.

\section{The case of one complex variable}

In the special case of $N=1$ the results of the previous sections can be improved considerably.

4.1. Lemma. For $N=1$ let $\mu \in A(K)^{\prime}$ such that $T_{\mu}: A(Q+K) \rightarrow A(Q)$ is surjective. Then $(\hat{\mu} \cdot A(\mathrm{C})) \cap A_{\bar{H}+\bar{L}}=\hat{\mu} \cdot A_{\bar{H}}$.

Proof. By Remark 1.9 (b), $A(Q)$ is ultrabornological. Since $A(Q+K)$ is a webbed space, it follows from de Wilde's theorem, that $T_{\mu}$ is even an open mapping. Hence $\operatorname{im} T_{\mu}^{\prime}=\left(\operatorname{ker} T_{\mu}\right)^{\perp}$ is closed in $A(Q+K)^{\prime}$. Since $T_{\mu}^{\prime}=$ $M_{\hat{\mu}}: A_{\bar{H}} \rightarrow A_{\bar{H}+\bar{L}}, f \mapsto \hat{\mu} f$, by Lemma 1.10 , the space $\hat{\mu} \cdot A_{\bar{H}}$ is closed in $A_{\bar{H}+\bar{L}}$.

On the other hand $\hat{\mu} \cdot A(\mathrm{C})$ is closed in $A(\mathrm{C})$. Since the embedding $A_{\bar{H}_{n}+\bar{L}} \rightarrow A\left(\mathrm{C}^{N}\right)$ is continuous for each $n \in \mathrm{N}$, also the embedding $A_{\bar{H}+\bar{L}} \rightarrow A\left(\mathrm{C}^{N}\right)$ is continuous. Thus also $(\hat{\mu} \cdot A(\mathrm{C})) \cap A_{\bar{H}+\bar{L}}$ is closed in $A_{\bar{H}+\bar{L}}$.

By Krasičkov-Ternovski [9], Thm. 4.4 (see [17], 1.6 (c)), $\hat{\mu} \cdot A_{\bar{H}}$ is dense in $(\hat{\mu} \cdot A(\mathrm{C})) \cap A_{\overline{H_{n}}+\bar{L}}$ for each $n \in \mathrm{N}$ and hence in $(\hat{\mu} \cdot A(\mathrm{C})) \cap A_{\bar{H}+\bar{L}}$. This proves the postulated identity.

Remark. In the case that $\hat{\mu}$ is slowly decreasing on $\mathbf{C}$ (for example if $K=\{0\}$ ), the conclusion of 4.1 follows simply dividing by $\hat{\mu}$ (and it does not matter whether $T_{\mu}$ is surjective or not). Of course, also the next Lemma is much simpler in this case.

4.2. Lemma. For $N=1$ let $\mu \in A(K)^{\prime}$ such that $T_{\mu}: A(Q+K) \rightarrow A(Q)$ is surjective. Let $A$ be a compact subset of $S$ having a neighborhood on which $\bar{H}$ is finite and such that $A$ is contained in the support of $\Delta \bar{H}$, i.e. for each $a \in A$ there is no neighborhood of a on which $\bar{H}$ is harmonic (and finite). Then $\hat{\mu}$ is slowly decreasing on $\Gamma(A)$.

Proof. Again by de Wilde's open mapping theorem, $T_{\mu}: A(Q+K) \rightarrow$ $A(Q)$ is an open mapping. Hence for each equicontinuous set $B \subset$ $A(Q+K)^{\prime}$, its preimage $\left(T_{\mu}^{\prime}\right)^{-1}(B)$ is equicontinuous again. Since obviously (see the proof of 1.9), $B \subset A(Q)^{\prime}$ is equicontinuous iff it is equicontinuous in 
some step $A\left(Q_{m}\right)^{\prime}$, by the Laplace transformation 1.9, we obtain the following condition:

If $f_{j}, j \in \mathrm{N}$, is a sequence in $A_{\bar{H}}$ and if there is $n$ such that for each $l$

$$
\sup _{z \in \mathrm{C}}\left|\hat{\mu}(z) f_{j}(z)\right| \exp \left(-H_{n}(\bar{z})-L(\bar{z})-|z| / l\right), \quad j \in \mathrm{N},
$$

is bounded, then there is some $m$ such that also

$$
\sup _{z \in \mathrm{C}}\left|f_{j}(z)\right| \exp \left(-H_{m}(\bar{z})-|z| / l\right), \quad j \in \mathrm{N},
$$

is bounded for each $l \in \mathrm{N}$. (See Meyer [18], Lemma 4.13, or [19], Lemma 3.12, for this kind of reasoning.)

We will now argue by contradiction applying a well known procedure of Ehrenpreis. We make use of some improvements made in [24].

If $\hat{\mu}$ is not slowly decreasing on $\Gamma(A)$, then there is some $k$ and a sequence $z_{j} \in \Gamma(A), j \in \mathrm{N}$, with $\left|z_{j}\right| \rightarrow \infty$ such that

$$
|\hat{\mu}(w)| \leq \exp (L(\bar{w})-|w| / k) \quad \text { for all }\left|w-z_{j}\right| \leq\left|z_{j}\right| / k, \quad j \in \mathrm{N} .
$$

For sufficiently large $k_{1}>k$ and $n$, and for each $j \in \mathrm{N}$ we consider the largest subharmonic function $p_{j}:=h\left(\bar{H}_{n}, z_{j},\left|z_{j}\right| / k_{1}\right)$ on $\mathrm{C}$ which equals $\bar{H}_{n}$ on $\left|z-z_{j}\right| \geq\left|z_{j}\right| / k_{1}$. Since $p_{j}$ is subharmonic, it is well known (see Hörmander [1], Thm. 4.4.4) that there is $f_{j} \in A(\mathrm{C})$ with $f_{j}\left(z_{j}\right)=\exp p_{j}\left(z_{j}\right)$ such that

$$
\left|f_{j}(z)\right| \leq C_{1} \exp \left(\tilde{p}_{j}(z)+C_{1} \log (1+|z|)\right), \quad z \in \mathrm{C},
$$

where $C_{1}>0$ is a universal constant and

$$
\tilde{p}_{j}(z):=\sup _{|w-z| \leq 1} p_{j}(w), \quad z \in \mathrm{C} .
$$

We obtain from the bound on $f_{j}$ that for some $C=C_{n}>0$

$$
\left|f_{j}(z)\right| \leq C \exp \left(H_{n}(\bar{z})+C_{1} \log (1+|z|)+\Delta\left(\bar{H}_{n}, z_{j},\left|z_{j}\right| / k_{1}+1\right)\right), \quad z \in \mathrm{C},
$$

where

$$
\Delta\left(\bar{H}_{n}, z_{j},\left|z_{j}\right| / k_{1}+1\right):=\max _{\left|z-z_{j}\right| \leq\left|z_{j}\right| / k_{1}+1}\left(h\left(\bar{H}_{n}, z_{j},\left|z_{j}\right| / k_{1}+1\right)(z)-\bar{H}_{n}(z)\right) .
$$

In particular, $f_{j} \in A_{\bar{H}_{n}}^{0} \subset A_{\bar{H}}$. Put $a_{j}:=z_{j} /\left|z_{j}\right|$. We may assume that $k_{1}$ is chosen so large that $\left|z_{j}\right| / k_{1}+1 \leq\left|z_{j}\right| / k$ and

$$
\Delta\left(\bar{H}_{n}, z_{j},\left|z_{j}\right| / k_{1}+1\right)=\left|z_{j}\right| \Delta\left(\bar{H}_{n}, a_{j}, 1 / k_{1}+1 /\left|z_{j}\right|\right) \leq\left|z_{j}\right| / k, \quad j \in \mathbf{N} .
$$

Because of the assumptions on $A$, the functions $H, H_{m}, m \in \mathrm{N}$, are equicontinuous on a neighborhood of $A$. Hence the choice of $k_{1}$ does not depend on $n$. We obtain 
$\left|\hat{\mu}(z) f_{j}(z)\right| \leq C \exp \left(H_{n}(\bar{z})+C_{1} \log (1+|z|)+L(\bar{z})\right) \quad$ for $\left|z-z_{j}\right| \leq\left|z_{j}\right| / k_{1}+1$.

Since $\hat{\mu} \in A_{\bar{L}}^{0}$, this implies that for each $l$ there is $C_{l} \geq C$ with

$$
\sup _{z \in \mathrm{C}}\left|\hat{\mu}(z) f_{j}(z)\right| \exp \left(-H_{n}(\bar{z})-L(\bar{z})-|z| / l\right) \leq C_{l} \quad \text { for all } j \in \mathrm{N} .
$$

Since $A$ is compact and $\bar{H}$ is finite on $A$, we can extend $\bar{H} \mid A$ to an appropriate continuous function in a neighborhood of $A$. Since $A \subset \operatorname{supp} \Delta \bar{H}$ this shows that there is some $n \in \mathrm{N}$ and some $k_{2}$ such that

$$
h\left(\bar{H}_{n}, a, 1 / k_{1}\right)(a)-\bar{H}_{n}(a)>1 / k_{2} \quad \text { for all } a \in A .
$$

Since $\bar{H} \mid A$ is continuous, $n$ can be chosen such that $h\left(\bar{H}_{n}, a_{j}, 1 / k_{1}\right)\left(a_{j}\right)-$ $\bar{H}\left(a_{j}\right) \geq 1 / k_{2}$ for all $j$. Hence

$$
f_{j}\left(z_{j}\right)=\exp \left(\left|z_{j}\right| h\left(\bar{H}_{n}, a_{j}, 1 / k_{1}\right)\right)\left(a_{j}\right) \geq \exp \left(H\left(\bar{z}_{j}\right)+\left|z_{j}\right| / k_{2}\right), \quad j \in \mathrm{N},
$$

and we obtain that for each $m \in \mathrm{N}$ there is $l=2 k_{2}$ such that

$$
\sup _{z \in \mathrm{C}}\left|f_{j}(z)\right| \exp \left(-H_{m}(\bar{z})-|z| / l\right) \geq \exp \left(\left|z_{j}\right| /\left(2 k_{2}\right)\right) \quad \text { for all } j \in \mathrm{N} .
$$

Obviously, this yields a contradiction.

4.3. Lemma. For $N=1$, assume that the multiplication operator $M_{\hat{\mu}}: A_{\bar{H}} \rightarrow$ $A_{\bar{H}+\bar{L}}$ admits a continuous linear left inverse. Then condition $U\left(A_{\hat{\mu}}, Q\right)$ holds.

Proof. If $A_{\hat{\mu}} \neq \emptyset$, we can choose an entire function $g \in A_{\{0\}}^{0}$, i.e. at most of order one and zero type, with zeros $\left(b_{l}\right)_{l \in \mathrm{N}}$ and with $A_{g}=A_{\hat{\mu}}$ and $\hat{\mu} / g \in A(\mathrm{C})$. Then also $M_{g}: A_{\bar{H}} \rightarrow A_{\bar{H}}$ has a continuous linear left inverse. Hence the canonical mapping $\rho: A_{\bar{H}} \rightarrow A_{\bar{H}}(g)$ has a continuous linear right inverse $R$ (Lemma 1.12 with $K=\{0\}$ ).

Let $\Omega(z), z \in \mathrm{C}$, be as in 2.5. We choose $f_{l} \in A_{H}(g)$ with $f_{l} \mid \Omega(z)=1$ $\bmod I(\Omega(z))$ if $b_{l} \in \Omega(z)$, and with $f_{l} \mid \Omega(z)=0 \bmod I(\Omega(z))$ if $b_{l} \notin \Omega(z)$.

We put $v_{l}:=\log \left|R\left(f_{l}\right)\right|, l \in \mathrm{N}$. Then $v_{l}\left(b_{l}\right) \geq 0, l \in \mathrm{N}$. Since $R: A_{\bar{H}}(g) \rightarrow$ $A_{\bar{H}}$ is continuous, by Grothendieck's factorization theorem, $\forall n \exists n^{\prime}$ such that $R: A_{\bar{H}_{n}}^{0}(g) \rightarrow A_{\bar{H}_{n^{\prime}}}^{0}$ is continuous, i.e. $\forall m \exists k, k_{1}, B, B_{1}>0$ with

$$
\sup _{z \in \mathrm{C}}\left(v_{l}(z)-H_{n^{\prime}}(z)-|z| / m\right) \leq B_{1}+\log \left\|f_{l}\right\|_{n, k_{1}} \leq B-H_{n}\left(b_{l}\right)-\left|b_{l}\right| / k, \quad l \in \mathrm{N} .
$$

Now if $a \in A_{g}$ with $a=\lim _{j \rightarrow \infty} b_{l_{j}} /\left|b_{l_{j}}\right|$, we put

$$
u_{a}(z):=\left(\limsup _{j \rightarrow \infty} \frac{v_{l_{j}}\left(z b_{l_{j}} / a\right)}{\left|b_{l_{j}}\right|}\right)^{*}, \quad z \in \mathrm{C},
$$

where ${ }^{*}$ denotes the upper semicontinuous regularization. Then $u_{a}$ is subharmonic with $u_{a}(a) \geq 0$. By straight forward estimation, we get the postulated upper bounds. 
4.4. Lemma. For $N=1$ and $\mu \in A(K)^{\prime}$ let $T_{\mu}: A(Q+K) \rightarrow A(Q)$ be surjective. If the condition $U\left(A_{\hat{\mu}}, Q\right)$ holds and $\bar{H}$ is finite on a neighborhood of $A_{\hat{\mu}}$, then $\hat{\mu}$ is slowly decreasing on $\Gamma\left(A_{\hat{\mu}}\right)=V_{\infty}(\hat{\mu})$.

Proof. By Remark 4.10, we know that $V_{\infty}(\hat{\mu})$ is contained in $\{t a \mid t \geq 0$, $a \in S, H(\bar{a})<\infty\} \cup \operatorname{supp} \Delta \bar{H}$. Hence, by Lemma 4.2, $\hat{\mu}$ is slowly decreasing on $V_{\infty}(\hat{\mu})$.

4.5. Proposition. For $N=1$ let $\mu \in A(K)^{\prime}$ such that $T_{\mu}: A(Q+K) \rightarrow$ $A(Q)$ is surjective. If $Q$ is bounded or $T_{\mu}=P(D)$ is a differential operator of infinite order, then $T_{\mu}: A(Q+K) \rightarrow A(Q)$ admits a continuous linear right inverse if and only if condition $U\left(A_{\hat{\mu}}, Q\right)$ holds.

Proof. $\Rightarrow$ : By Lemma 4.3 and Lemma 1.12.

$\Leftarrow$ : By Lemmas 4.1 and 4.4, the hypotheses of Proposition 2.7 are fulfilled. Hence by Proposition 2.7 and Lemmas 1.10 and 1.12, the map $M_{\hat{\mu}}: A_{\bar{H}} \rightarrow A_{\bar{H}+\bar{L}}$ admits a continuous linear left inverse. Thus the assertion follows from Lemma 1.12.

4.6. Proposition. For $N=1$, assume that 0 is contained in the relative interior of $Q$. Each nonzero differential operator $P(D): A(Q) \rightarrow A(Q)$ has a continuous linear right inverse if and only if either $Q=\mathrm{C}$ or if $Q$ is strictly convex at $\partial_{r} \omega$, is bounded, has nonempty interior, such that $C_{H}^{\infty}$ is bounded on a neighborhood of $S_{o}$ in $S$, and $1 / C_{H}^{0}$ is bounded on each compact subset of $S_{\omega}$.

In this case even each surjective convolution operator $T_{\mu}: A(Q+K) \rightarrow A(Q)$ has a continuous linear right inverse.

Proof. The first part of the assertion follows immediately from Propositions 1.15 and 1.16, and from Proposition 3.10 and Theorem 3.9.

If $Q=\mathrm{C}$, each convolution operator admits a continuous linear right inverse, by Schwerdtfeger [33] and Taylor [34] (see also [23]).

If $Q$ is bounded with nonempty interior and strictly convex at $\partial_{r} \omega$, then each surjective $T_{\mu}$ admits a continuous linear right inverse by Propositions 4.5 and 3.6.

4.7. Proposition. For $N=1$, assume that $Q$ is strictly convex at $\partial_{r} \omega$, is bounded, with 0 in its nonempty interior. Then a given surjective convolution operator $T_{\mu}: A(Q+K) \rightarrow A(Q)$ has a continuous linear right inverse if and only if $C_{H}^{\infty}$ is bounded on a neighborhood of $A_{o}:=\overline{A_{\hat{\mu}}} \cap S_{o}$ in $\bar{A}$ and $1 / C_{H}^{0}$ is bounded on each compact subset of $A_{\omega}:=\overline{A_{\hat{\mu}}} \cap S_{\omega}$.

Proof. By Propositions 4.5 and 3.6.

For $N=1$, in some special cases, we are going to prove a charactarization 
of the existence of a right inverse also if $Q$ is not bounded, or has empty interior, or is not strictly convex at $\partial_{r} \omega$.

4.8. Definition. Let $a \in \mathrm{C}^{N} \backslash\{0\}$. A plurisubharmonic function $v$ defined on a neighborhood of $a$ is called a plurisubharmonic saddle for $H$ at $a$ if $v \leq H, v(a)=H(a)$, and $v(z)<H(z)$ whenever $z \in \Gamma(a) \backslash\{a\} . \quad v$ is called proper if even $v(z)<H(z)$ whenever $z \in \mathrm{C}^{N} \backslash\{a\}$. (See [26] and [28].)

4.9. Proposition. For $N=1$, let $Q \neq \mathrm{C}$ and $A \subset S$ with $\# A<\infty$. Then the condition $U(A, Q)$ holds if and only if for each $a \in A$ we have $H(\bar{a})<\infty$ and there is a proper subharmonic saddle for $H$ at $\bar{a}$.

Proof. For the proof we may assume that $A=\{a\}$.

" $\Rightarrow$ ". By condition $U(A, Q)$, there is a subharmonic function $u$ on $\mathrm{C}$ with $u(a) \geq 0$ and $\forall n \exists n^{\prime} \forall m \exists k$ such that

$$
u \leq \bar{H}_{n^{\prime}}+|\cdot| / m-H_{n}(\bar{a})-1 / k .
$$

Note that $\bar{u}(z):=u(\bar{z})$ is again subharmonic, $\bar{u}(\bar{a}) \geq 0$, and $\forall n \exists n^{\prime} \forall m \exists k$ such that

$$
\bar{u} \leq H_{n^{\prime}}+|\cdot| / m-H_{n}(\bar{a})-1 / k .
$$

Hence for each $n$ there is $n^{\prime}$ with $\bar{u} \leq H_{n^{\prime}}-H_{n}(\bar{a})$, which implies $H(\bar{a})<\infty$; otherwise, if $z^{0}$ is chosen with $H\left(z^{0}\right)<\infty$, we could conclude that $\bar{u}\left(t z^{0}\right) \leq H\left(t z^{0}\right)-H(\bar{a})=-\infty$ for all $t>0$, which is impossible since $] 0, \infty[$ is not polar.

We define $v:=\bar{u}+H(\bar{a})$. Then $v(\bar{a}) \geq H(\bar{a})$, and as above we obtain $v \leq H$, and thus $v(\bar{a})=H(\bar{a})$.

Let $\bar{a} \in S_{o}$ : (3) applied to $n=1$ gives some $n^{\prime}$. By the hypothesis we can choose $m$ such that $H_{n^{\prime}}(\bar{a})+|a| 2 / m<H(\bar{a})$. Hence there is even an open neigborhood $A^{\prime}$ of $\bar{a}$ in $S$ such that $H_{n^{\prime}}+|\cdot| 2 / m \leq H$ holds on the cone $\Gamma\left(A^{\prime}\right)$. If $k$ is chosen according to (3), we obtain with some $C>0$ that

$v(z) \leq H_{n^{\prime}}(z)+|z| / m+H(\bar{a})-H_{1}(\bar{a})-1 / k \leq H(z)-|z| / m+C, \quad z \in \Gamma\left(A^{\prime}\right)$.

Let $L$ be an R-linear function with $L \leq H$ and $L(a)=H(a)$. Then $L=H$ precisely on a convex cone $\Gamma_{L}$. If $w \in \Gamma\left(A^{\prime}\right)$ and $v(w)=H(w)$, then $w \notin \operatorname{int} \Gamma_{L}$; otherwise $v-L$ would be (by the preceding estimate for large $|z|$ ) a non constant subharmonic function attaining its maximum in an inner point of its domain of definition. This shows that there is a neighborhood $\tilde{A} \subset A^{\prime}$ of $a$ and some $R>1$ such that

$$
\tilde{v}:=v / 2+L / 2<H \text { on } \Gamma(\tilde{A}) \backslash([0, R] \cdot\{\bar{a}\}),
$$


$\tilde{v} \leq H$, and $\tilde{v}(\bar{a})=H(\bar{a})$. Since $\tilde{v}$ is subharmonic, as in [26], Prop. 5, this implies that there is a proper subharmonic saddle for $H$ at $a$.

Let $\bar{a} \in S_{\omega}$ : Then $H_{n}(\bar{a})=H(\bar{a})$ for some $n$. By (3) $\exists n^{\prime} \forall m \exists k$ with

$$
v \leq H_{n^{\prime}}+|\cdot| / m+H(\bar{a})-H_{n}(\bar{a})-1 / k=H_{n^{\prime}}+|\cdot| / m-1 / k .
$$

In particular $v(0) \leq-1 / k<0(m=1)$, and $v$ is even bounded by $H_{n^{\prime}}$, not only by $H$. By [26], Prop. 5, this implies that there is a saddle for $H_{n^{\prime}}$ and hence for $H$ at $\bar{a}$. As in the case " $\bar{a} \in S_{o}$ " one can modify $v$ to become a proper saddle.

" $\Leftarrow$ ": Let $\bar{a} \in S_{o}$ : If $u: D \rightarrow \mathrm{R}$ is a proper subharmonic saddle for $H$ at $\bar{a}$ defined on a open bounded neighborhood of $\bar{a}$, we can choose $p$ so large that $u<H_{p}$ on $\partial D$. We consider the subharmonic function $v:=\max \left\{u, H_{p}\right\}$ on $D$ and $v:=H_{p}$ on $\mathrm{C} \backslash D$. It satisfies $v(\bar{a})=H(\bar{a})$ and $v \leq H$. We claim that $\forall n \exists n^{\prime}$ with

$$
v \leq H_{n^{\prime}}+1 / n
$$

This is obvious on $\mathrm{C} \backslash D$. Since $u-1 / n<H$ on $D$, there is $n^{\prime}$ such that $u-1 / n<H_{n^{\prime}}$ there. This proves the claim.

Now, if an arbitrary $n$ is given, because of $\bar{a} \in S_{o}$, we choose $\tilde{n}$ with $1 / \tilde{n} \leq\left(H(\bar{a})-H_{n}(\bar{a})\right) / 2$. Next choose $n^{\prime}$ with $v \leq H_{n^{\prime}}+1 / \tilde{n}$. We obtain for all $m$ and for $k$ with $1 / k \leq\left(H(\bar{a})-H_{n}(\bar{a})\right) / 2$

$$
\begin{aligned}
v & \leq H_{n^{\prime}}+1 / \tilde{n} \\
& \leq H_{n^{\prime}}+H(\bar{a})-H_{n}(\bar{a})-1 / k \\
& \leq H_{n^{\prime}}+|\cdot| / m+H(\bar{a})-H_{n}(\bar{a})-1 / k .
\end{aligned}
$$

Let $\bar{a} \in S_{\omega}$ : The supporting line $\operatorname{Re}\langle z, \bar{a}\rangle=H(\bar{a})$ to $\operatorname{cl} Q$ touches $\partial Q$ in some point $w$ of $\omega$. If $w$ is an exposed point of $\operatorname{cl} Q$, then (since $\omega$ is open in $\partial_{r} Q$ ) there is $n^{\prime}$ with $H_{n^{\prime}}=H$ on a neighborhood of $\bar{a}$. Otherwise the supporting line contains even an open line segment of $\omega$. As in Proposition 3.6 one shows that for sufficiently large $n^{\prime}$ with $H_{n^{\prime}}(\bar{a})=H(\bar{a})$, there is a proper subharmonic saddle for some $H_{n^{\prime}}$ at $\bar{a}$. Hence by the hypothesis, in both cases there is a proper subharmonic saddle for $H_{n^{\prime}}$ at $\bar{a}$. By [26], Prop. 5, we have $C_{H_{n^{\prime}}}^{0}(\bar{a})>0$. By [17], Lemma 2.9, there is a subharmonic function $v$ on $\mathrm{C}$ with $v \leq H_{n^{\prime}}, v(\bar{a})=H_{n^{\prime}}(\bar{a})$, and such that $\forall m \exists k$ with

$$
v \leq H_{n^{\prime}}+|\cdot| / m-1 / k .
$$

Now, if $n$ is given, for each $m$ we choose $k$ satisfying the preceding estimate and obtain

$$
v \leq H_{n^{\prime}}+|\cdot| / m+H(\bar{a})-H_{n}(\bar{a})-1 / k .
$$

Finally, in both cases we define $u_{a}:=\bar{v}-H(\bar{a})$, and the proof is finished. 
4.10. Remark. For $N=1$ and $a \in S$ assume that the condition $U(\{a\}, Q)$ holds. Then $H(\bar{a})<\infty$ and there is no neighborhood of $\bar{a}$ on which $H$ is harmonic.

Proof. By Proposition 4.9, $H(\bar{a})<\infty$. If $H$ is harmonic on a neighborhood of $\bar{a}$, it follows from the maximum principle that there is no subharmonic saddle for $H$ at $\bar{a}$.

In view of Proposition 4.5, the following example contains some of the main results of Langenbruch [12] and Korobeĭnik [6]:

4.11. Example. For $N=1$ let $Q \subset \mathrm{C}$ be a polyhedron, i.e. $\mathrm{cl} Q$ is the intersection of halfplanes $\left\{w \in \mathrm{C} \mid \operatorname{Re}\langle w, a\rangle \leq H_{a}\right\}, H_{a} \in \mathrm{R}, a \in A$, where $A \subset S$ is a finite set. We assume that none of these halfplanes is superflous in this representation of $\mathrm{cl} Q$. Then condition $U\left(A_{\hat{\mu}}, Q\right)$ is fulfilled if and only if $\overline{A_{\hat{\mu}}}:=\left\{\bar{a} \mid a \in A_{\hat{\mu}}\right\} \subset A$.

Proof. " $\Rightarrow$ ": Let $a \in A_{\hat{\mu}}$. By Proposition 4.9, $H(\bar{a})<\infty$. Hence $\bar{a} \in A$ or $H$ is R-linear (hence harmonic) in some neighborhood of $\bar{a}$. Again by Proposition 4.9, there is subharmonic saddle for $H$ at $\bar{a}$. Thus, by Remark 4.10, the latter case cannot occur.

" $\Leftarrow$ ": If $a \in A$ we have $H(a)=H_{a}<\infty$. For a suitable R-linear function $L$ on $\mathrm{C}$ and some $c>0$, we obtain after an appropriate rotation and translation of $\mathrm{C}$ that $H(z)-L(z) \geq c|\operatorname{Im} z|$ in a neighborhood of 1 , and $H(1)-L(1)=0$. For $z \mapsto|\operatorname{Im} z|$ there is a proper subharmonic saddle at 1 (for instance $\left.z \mapsto(\operatorname{Im} z)^{2}-(\operatorname{Re} z-1)^{2}\right)$, thus there is also one for $H$ at $\bar{a}$. Hence the assertion follows from Proposition 4.9.

\section{REFERENCES}

1. L. Hörmander, An Introduction to Complex Analysis in Several Variables, Princeton 1967.

2. L. Hörmander, On the existence of real analytic solutions of partial differential equations with constant coefficients, Invent. Math. 21 (1973), 151-182.

3. L. Hörmander, Notions of convexity, Birkhäuser Boston, Basel, Berlin 1994.

4. Ju. F. Korobeĭnik, The existence of an analytic solution of an infinite order differential equation and the nature of its domain of analyticity, Math. USSR-Sb. 9 (1969) 53-71.

5. Ju. F. Korobeĭnik, Solvability of a convolution equation in some classes of analytic functions, Math. Notes 49 (1991), 165-172.

6. Ju. F. Korobeĭnik, Convolution operators on spaces of analytic germs in C, Linear Topological Spaces and Complex Analysis 1 (1994), 33-51.

7. Ju. F. Korobeĭnik, On a linear right inverse for convolution operators on spaces of germs on connected sets in C, Mat. Sb. 187, No. 1 (1996), 55-82 (Russian).

8. Ju. F. Korobeĭnik \& S. N. Melikhov, A linear continuous right inverse for the representation operator and an application to convolution operators, Sibirsk. Mat. J. 34, No. 1, (1993), 70-84 (Russian). 
9. I. F. Krasičkov-Ternovski, Invariant subspaces of analytic functions I, Math. USSR-Sb. 16 (1972), 471-500.

10. A. S. Krivosheev, A criterion for the solvability of nonhomogeneous convolution equations in convex domains of $\mathrm{C}^{N}$, Math. USSR-Izv. 36 (1991), 497-517.

11. M. Langenbruch, Splitting of the $\bar{\partial}$-complex in weighted spaces of square integrable functions, Rev. Mat. Univ. Complut. Madrid 5 (1992), 201-223.

12. M. Langenbruch, Continuous linear right inverses for convolution operators in spaces of real analytic functions, Studia Math. 110 (1994), 65-82.

13. I. M. Maltsev, Surjectivity of a convolution operator in spaces of analytic functions on connected sets, Russian Acad. Sci. Dokl. Math. (former Soviet Math. Dokl.) 49 (1994), 501-506.

14. A. Martineau, Sur la topologie des espaces de fonctions holomorphes, Math. Ann. 163 (1966), 62-88.

15. A. Martineau, Équations différentielles d'ordre infini, Bull. Soc. Math. France 95 (1967), 109-154.

16. R. Meise \& B. A. Taylor, Each non-zero convolution operator on the entire functions admits a continuous linear right inverse, Math. Z. 197 (1988), 139-152.

17. S. Melikhov \& S. Momm, Solution operators for convolution equations on the germs of analytic functions on compact convex sets of $\mathrm{C}^{N}$, Studia Math. 117 (1995), 79-99.

18. T. Meyer, Surjektivität von Faltungsoperatoren auf Räumen ultradifferenzierbarer Funktionen vom Roumieu-Typ. Thesis, Düsseldorf 1992.

19. T. Meyer, Surjectivity of convolution operators on spaces of ultradifferentiable functions of Roumieu type, Studia Math. 125 (1997), 101-129.

20. S. Momm, Convex univalent functions and continuous linear right inverses, J. Funct. Anal. 103 (1992), 85-103.

21. S. Momm, Division problems in spaces of entire functions of finite order, In Functional Analysis, Bierstedt, Pietsch, Ruess, Vogt (eds.), Marcel Dekker New York 1993, 435457.

22. S. Momm, A critical growth rate of the pluricomplex Green function, Duke Math. J. 72 (1993), 487-502.

23. S. Momm, Convolution equations on the analytic functions on convex domains in the plane, Bull. Sci. Math. 118 (1994), 259-270.

24. S. Momm, A division problem in the space of entire functions of exponential type, Ark. Mat. 32 (1994), 213-236.

25. S. Momm, The boundary behavior of extremal plurisubharmonic functions, Acta Math. 172 (1994), 51-75.

26. S. Momm, Plurisubharmonic saddels, Ann. Polon. Math. 63 (1996), 235-245.

27. S. Momm, An extremal plurisubharmonic function associated to a convex pluricomplex Green function with pole at infinity, J. Reine Angew. Math. 471 (1996), 139-163.

28. S. Momm, Extremal plurisubharmonic functions for convex bodies in $\mathrm{C}^{N}$, In Complex analysis, harmonic analysis and applications, Deville, R. (ed.) et al. Bordeaux, France 1995, Pitman Res. Notes Math. Ser. 347 (1996), 87-103.

29. V. V. Morzhakov, On epimorphicity of a convolution operator in convex domains in $\mathrm{C}^{N}$, Math. USSR-Sb. 60 (1988), 347-364.

30. V. V. Napalkov \& I. A. Rudakov, Convolution operators in the space of real analytic functions, Math. Notes 49 (1991), 57-65.

31. H. Schaefer, Topological Vector Spaces, Macmillan, New York, 1966.

32. R. Schneider, Convex Bodies, The Brunn-Minkowsky Theory, Cambridge University Press, 1993.

33. K. Schwerdtfeger, Faltungsoperatoren auf Räumen holomorpher und beliebig oft differenzierbarer Funktionen, Thesis, Düsseldorf 1982.

34. B. A. Taylor, Linear extension operators for entire functions, Michigan Math. J. 29 (1982), 185-197. 
35. D. Vogt, Topics on projective spectra of (LB)-spaces, in Advances in the theory of Fréchet spaces, T. Terzioğlu (Ed.), Istanbul 1987, NATO ASI Series C, Vol. 287, Kluwer, Dordrecht 1989, 11-27.

36. J. Wengenroth, Retractive (LF)-Spaces, Thesis, Trier 1995.

37. J. Wengenroth, Acyclic inductive spectra of Fréchet spaces, Studia Math. 120 (1996), 247258.

DEPARTMENT OF MECHANICS AND MATHEMATICS ROSTOV STATE UNIVERSITY

ZORGE ST. 5

344090 ROSTOV ON DON

RUSSIA
MATHEMATISCHES INSTITUT

HEINRICH-HEINE-UNIVERSITÄT DÜSSELDORF

UNIVERSITÄTSSTRAßE 1

40225 DÜSSELDORF

GERMANY 